\title{
Relic density of dark matter in the inert doublet model beyond leading order for the low mass region. I. Renormalization and constraints
}

\author{
Shankha Banerjee $\odot,{ }^{1, *}$ Fawzi Boudjema $\odot,{ }^{2, \dagger}$ Nabarun Chakrabarty, ${ }^{3,4, \star}$ and Hao Sun ${ }^{5, \S}$ \\ ${ }^{1}$ CERN, Theoretical Physics Department, CH-1211 Geneva 23, Switzerland \\ ${ }^{2}$ LAPTh, Université Savoie Mont Blanc, CNRS, BP 110, F-74941 Annecy-le-Vieux, France \\ ${ }^{3}$ Centre for High Energy Physics, Indian Institute of Science, \\ C.V. Raman Avenue, Bangalore 560012, India \\ ${ }^{4}$ Department of Physics, Indian Institute of Technology Kanpur, Kanpur, Uttar Pradesh 208016, India \\ ${ }^{5}$ Institute of Theoretical Physics, School of Physics, Dalian University of Technology, \\ Dalian 116024, People's Republic of China
}

(Received 20 February 2021; accepted 13 August 2021; published 5 October 2021)

The present paper is the first in a series that addresses the calculation of the full one-loop corrections of dark matter (DM) annihilation cross sections in the low mass region of the inert doublet model (IDM). This series is a sequel to our recent publication concerning these corrections in the high mass region. We first review the renormalization of the model both in a fully on shell (OS) scheme as well as in a mixed scheme that combines on shell (for the masses) and a $\overline{\mathrm{MS}}$ approach when the partial invisible width is closed and does not allow the use of a full OS scheme. The scale dependence introduced by the mixed scheme is shown to be tracked through an analysis of a parametrization of the tree-level cross section and the $\beta$ constant of a specific coupling; this analysis could be followed in theories. We discuss how to minimize the scale dependence. The theoretical uncertainty brought by the scale dependence leads us to introduce a new criterion on the perturbativity of the IDM. This criterion further delimits the allowed parameter space, which we investigate carefully by including a host of constraints, both theoretical and experimental, including in particular, new data from the LHC. We come up with a set of benchmark points that cover three different mechanisms for a viable relic density of DM: (i) a dominance of coannihilation into a fermion pair, (ii) annihilation into two vector bosons of which one is off shell that requires the calculation of a $2 \rightarrow 3$ process, (iii) annihilation that proceeds through the very narrow standard model Higgs resonance. Since the $2 \rightarrow 3$ vector boson channel features in all three channels and is essentially a buildup on the simpler annihilation to OS vector bosons, we study the latter in detail in the present paper. We confirm again that the corrected cross sections involve a parameter that can be considered as rescattering in the dark sector, which a tree-level computation is not sensitive to. The setup of the renormalization detailed in the present paper will be the backbone of the accompanying papers where each mechanism requires, calculationally, a specific treatment. One-loop corrections to $2 \rightarrow 3$ processes for DM annihilation are technically challenging and have not been attempted before. We dedicate one of the accompanying papers to such a computation. The one-loop correction in the presence of a resonance will be presented separately since we need to supplement our general schemes with a complex scheme. For the coannihilation into fermions, our study will show that the annihilation cross sections can be excellently parametrized through simple effective couplings.

DOI: 10.1103/PhysRevD.104.075002

\footnotetext{
*shankha.banerjee@cern.ch

†boudjema@lapth.cnrs.fr

*chakrabartynabarun@gmail.com

§haosun@dlut.edu.cn
}

Published by the American Physical Society under the terms of the Creative Commons Attribution 4.0 International license. Further distribution of this work must maintain attribution to the author(s) and the published article's title, journal citation, and DOI. Funded by SCOAP.

\section{INTRODUCTION}

Cosmology has entered the era of precision measurements. One such measurement is the inferred relic density of dark matter (DM) that is now determined at the percent level [1]. For a particle physicist, such an accuracy is reminiscent of the one achieved at Large Electron Positron Collider (LEP). In turn, on the theory side, this level of accuracy requires that observables be computed with a precision on par with the experimental precision or even better. Given the cosmological model for the thermodynamics/evolution of 
the Universe and a model of DM, the computation of the relic density of DM involves the calculations of annihilation rates. While, unfortunately, no sign of a model of DM has emerged either in direct detection or at the colliders, many models have been proposed, and a huge amount of work has been dedicated to the search and study of these models. Yet, despite the central role that the very precise measurement of the relic density plays in constraining the phenomenology of these models, only a few examples have provided the calculation of the annihilation rates beyond the leading order, tree-level approximation. Most of these examples concerned the minimal supersymmetric standard model (MSSM), including full electroweak [2-10] and QCD corrections [3,11-17]. Sommerfeld effects [18-29] and Sudakov effects [30-33] that are of importance for $\mathrm{TeV}$ DM annihilation, especially for indirect detection [2,34-40], have been computed for a variety of models. Next-to-leading order (NLO) corrections to DM direct detection have been calculated in some cases [41-51]. In this series of papers, we will tackle the computation of many annihilation rates that occur in different viable scenarios of the inert double model (IDM) [52-54], in particular the low mass DM region in this model, beyond the tree-level approximation. This is a continuation of the work we initiated in [10] to cover the heavy DM scenario, $500 \mathrm{GeV}$ to $1 \mathrm{TeV}$, which complements important nonperturbative electroweak Sommerfeld effects [18-20,23,29] that are relevant beyond the TeV scale. As far as corrections affecting the annihilation cross sections are concerned, let us add a few words about (possible) temperature corrections. It has been shown quite sometime ago [55] that the temperature corrections are totally negligible, at least in the freeze-out scenario that will be our main application. This issue has been reassessed [56,57] more recently in conjunction with infrared divergences. The latter not only cancel at a finite temperature, but their thermal effect is again totally negligible, so that we only need to make sure that infrared divergences cancel at zero temperature as we will do in this study.

The IDM, with its possible link between the Higgs sector and DM [54], has enjoyed some popularity [23,46,58-93], but its structure beyond the tree level has been looked at only for some specific applications or conditions [46,51, 54,75,94-106]. In order to perform one-loop calculations, for any process in the IDM, and in particular, for DM annihilation into standard model (SM) particles, we need a full and coherent renormalization program for the IDM. Here, we present the details of our renormalisation schemes. We underline many important features that we believe will be very useful not only in studying DM annihilation in the IDM but also in studying the renormalization of models beyond the standard model (BSM).

Having obtained the one-loop corrected, velocity dependent, annihilation cross sections, we will need to convert them into a prediction for the relic density. This conversion will be carried out in the context of the freeze-out scenario.
We will interface our improved rates with the widely used micrOMEGAs code [107], where the numerical accuracy of the convolution is better than the percent, given sufficient values of the cross sections across the relative velocity range. We stress that the aim of our study is to give as precise theoretical predictions of the annihilation cross sections, and subsequently, to the relic density, assuming the freeze-out mechanism.

Independently of the size of the corrections of the oneloop calculation of the cross sections and, as we will see, the interesting new indirect effects that the latter brings, the necessity of a renormalization program forces one to give a physical definition to the minimal set of (independent) input parameters that define model. This elevates some parameters, from mere parameters at the Lagrangian level to physical input parameters defined from physical observables, generally defined at some scale whose dependence we can study. The theoretical uncertainty associated to the scale choice is another facet of the uncertainty that arises from the parametric uncertainty. The latter derives from the uncertainty on the value of the input parameters even if the latter were reconstructed from, say collider, experimental observables. A scan on the parameters of the Lagrangian, as has been the tradition for tree-level analyses of new physics models, usually projected on a map, does not provide a meaningful measure of such uncertainties. Improving the theoretical precision and finding how large these corrections can be is one aim of this series. This goes hand in hand with setting up and developing the techniques to make such precision calculations possible as we will do here and in the next articles in the series, in more detail. Of course, as with any theory, and more so for a model of new physics, the inherent parametric uncertainty is important: a change in a parameter will shift the value of the relic density for example. A strategy of how precisely some key parameters need to be measured to match the precision of the relic density has been laid out by one of us in [108] in the framework of the MSSM. This strategy can be trivially adopted for the IDM. Considering the present precision on the relic density and precision from future runs of the LHC (and from possibly other facilities), such studies require theoretical precision calculations. Take the SM alone, at tree level, it predicts the same value for the angle $\left(\sin ^{2} \theta_{W}\right)$ derived from the mass ratio of weak neutral and charged weak bosons as the one derived from the asymmetries at $Z$ peak. Radiative corrections change that relation dramatically. Likewise, as will be shown in this series, the parameter dependence for the one-loop improved analyses of the relic density is larger than that based on tree-level calculations; a crucial parameter of the IDM model only appears at one-loop. This indirect effect is akin to the indirect dependence of LEP observables on the top and Higgs masses. These theoretical indirect effects will be qualitatively weighed. Radiative corrections, independent of their size, to the relic density that we will conduct in the 
IDM, is a first necessary step to study many of these interesting aspects.

As has only recently been uncovered, the small (DM) mass region consists in fact of three subregions, whose characteristics we confirm in this study. The main production mechanism is quite different in these three-regions which, at the one-loop level, call for rather different and sophisticated computational techniques. Since these techniques are rather involved, the details of the calculations and the discussions are kept separate in three accompanying papers. To unravel the one-loop features, for each one of these subregions, we perform the full one-loop electroweak corrections for two or three benchmark points chosen in each subregion in order to illustrate the fact that our results generalize to other benchmarks of the subregions. Still, for those wishing to perform a one-loop calculation for scattering processes in the IDM, we provide the necessary model files. The corrected cross sections, either those we have studied in these series with the freeze-out mechanism, could then be interfaced with micrOMEGAs or any alternative code for conversion of the annihilation rates to DM observables or in conjunction with colliders observables.

The plan of this first, parent paper in the series is as follows. In the next section, Sec. II, we briefly describe the model. This will bring forth the meaning of the physical parameters (rather than the parameters of the underlying Lagrangian). This will then set the stage to Sec. III on renormalization where we strive as much as possible to take an on shell (OS) scheme, which will use the physical masses of the model and the partial width of the SM Higgs boson to a pair of DM. Even in the narrow range of masses for the IDM that we will study here, a fully OS scheme is not possible when the latter's partial width is kinematically closed. In this case, we advocate a mixed OS- $\overline{\mathrm{MS}}$ scheme. This mixed scheme introduces a scale dependence, but we will show, on a simple example first, how to track the scale dependence through the $\beta$ constant of the associated coupling and a knowledge of the tree-level dependence on this coupling. This section will give us the opportunity to present our automated code to conduct one-loop calculations and the means we have to check the correctness of the results (switching between different choices of nonlinear gauge-fixing parameters [3,109] and ultraviolet finiteness of the cross sections and decays). Section IV is lengthy but necessary since we reanalyze all available experimental data and theoretical arguments to delimit the new parameter space of the model. The constraints include new data on direct detection and also new data from the LHC. We then propose a set of benchmark points, which will be scrutinized further by computing their respective relic density based on one-loop cross sections. We will see how the relic density predictions compare with those derived with tree-level cross sections. The thorough scan of the parameter space reveals in fact three mechanisms that permit a good DM candidate with a mass below the $W$ boson's, $M_{W}$. The features of these mechanisms are summarized in Sec. V. They consist of (i) a narrow coannihilation region driven essentially by gauge coupling, (ii) annihilations driven essentially by the SM Higgs resonance, and (iii) annihilations into three-body final states, $W f \bar{f}^{\prime}, Z f \bar{f}$ built up (mainly) on $W W^{\star}, Z Z^{\star}$ $\left(W^{\star}, Z^{\star}\right.$ denote the off shell vector bosons, a notion which will become clearer when we study the $2 \rightarrow 3$ processes). The technicalities involved in these three mechanisms are quite different, and this is the reason we decided to present the calculations in these three cases in three separate publications for more clarity and readability. In particular, (ii) and (iii) are very challenging. This is the first time that annihilation of DM to three particles is conducted at one loop. Renormalization and loop corrections in the presence of a resonance require extreme care; in fact, our paper [110] on the mechanism of annihilation through a Higgs can serve as a good example for many other BSM processes. Very subtle issues about renormalization beyond what is presented in the parent paper of the series will be highlighted in [110]. Moreover, even in the so-called coannihilation scenario [111], there is a small (but non-negligible) contribution that proceeds through annihilation to threebody final state. A contribution from $W f \bar{f}^{\prime}$ will also feature in the Higgs resonance mechanism. This is one of the reasons why, before tackling the challenging $W f \bar{f}^{\prime}, Z f \bar{f}$ in [112], the present paper studies in Sec. VII, as a warm-up, the annihilation to a pair of on shell $W^{+} W^{-}$and $Z Z$, even if phenomenologically they lead to underabundance. Another reason is that in this mass range, a fully OS scheme is not possible, and we need to use the mixed scheme. We therefore study the scale dependence of these annihilation cross sections and suggest an optimal scale for such processes thereby putting a conjecture, we make for the Higgs decay in this paper and for other processes elsewhere, on more solid ground. This study will also reveal that certain choices of parameters lead to too large scale dependence and even a breakdown of perturbativity in the sense of the loop expansion. We propose a criterion to avoid such configurations, a criterion that goes beyond the perturbativity argument used in our constraints in Sec. IV. This helps reduce the number of the benchmark points we use in the accompanying papers of this series. In Sec. VI, we go over how our calculations are set up by going through the different steps and interfaces as well as how we test the correctness of our computation. This will apply also to the detailed calculations we perform in the accompanying papers. We end the present paper with a short conclusion in Sec. VIII.

\section{THE MODEL AND THE PARAMETERS}

The IDM consists, in addition to the Standard Model (SM) Higgs doublet $\Phi_{1}$, of an extra doublet of scalars $\Phi_{2}$ on which a discrete $\mathbb{Z}_{2}$ symmetry is imposed. This 
symmetry entails that $\Phi_{2}$ is odd while all other fields (of the $\mathrm{SM})$ are even. As a consequence, this symmetry guarantees the stability of the lightest of the scalars of the $\Phi_{2}$ doublet. If the latter is neutral, it qualifies as a possible dark matter candidate. Another important upshot of this symmetry is that these extra scalar fields in $\Phi_{2}$ cannot couple to fermions, at least through renormalizable operators. Keeping only renormalizable operators, the scalar sector is therefore modified to

$\mathcal{L}_{\mathrm{IDM}}^{\text {scalar }}=\left(D^{\mu} \Phi_{1}\right)^{\dagger} D_{\mu} \Phi_{1}+\left(D^{\mu} \Phi_{2}\right)^{\dagger} D_{\mu} \Phi_{2}-\mathcal{V}_{\mathrm{IDM}}\left(\Phi_{1}, \Phi_{2}\right)$,

with

$$
\begin{aligned}
\mathcal{V}_{\mathrm{IDM}}\left(\Phi_{1}, \Phi_{2}\right)= & \mu_{1}^{2}\left|\Phi_{1}\right|^{2}+\mu_{2}^{2}\left|\Phi_{2}\right|^{2}+\lambda_{1}\left|\Phi_{1}\right|^{4}+\lambda_{2}\left|\Phi_{2}\right|^{4} \\
& +\lambda_{3}\left|\Phi_{1}\right|^{2}\left|\Phi_{2}\right|^{2}+\lambda_{4}\left(\Phi_{2}^{\dagger} \Phi_{1}\right)\left(\Phi_{1}^{\dagger} \Phi_{2}\right) \\
& +\left(\frac{\lambda_{5}}{2}\left(\Phi_{1}^{\dagger} \Phi_{2}\right)^{2}+\text { H.c. }\right) .
\end{aligned}
$$

$\mu_{i}$ and $\lambda_{i}$ are real and $D_{\mu}$ is the covariant derivative.

We parameterize the doublets as

$\Phi_{1}=\left(\begin{array}{c}G^{+} \\ \frac{1}{\sqrt{2}}\left(v+h+i G^{0}\right)\end{array}\right)$ and $\Phi_{2}=\left(\begin{array}{c}H^{+} \\ \frac{1}{\sqrt{2}}(X+i A)\end{array}\right)$,

where $v$ is the SM vacuum expectation value (vev) with $v \simeq 246 \mathrm{GeV}$, defined from the measurement of the $W$ $\left(M_{W}\right)$ and $Z\left(M_{Z}\right)$ masses. We have

$s_{W}^{2} \equiv \sin ^{2} \theta_{W}=1-\frac{M_{W}^{2}}{M_{Z}^{2}}, \quad M_{W}=\frac{1}{2} \frac{e}{s_{W}} v, \quad\left(v=\frac{2 M_{W} s_{W}}{e}\right)$,

where $e$ is the electromagnetic coupling. The $S U(2)$ gauge coupling, $g$, and the hypercharge gauge coupling, $g^{\prime}$, are then

$$
g=e / s_{W}, \quad g^{\prime}=e / c_{W} .
$$

$h$ is the SM Higgs boson (with a mass $M_{h}=125 \mathrm{GeV}$ ), and $G^{0}, G^{ \pm}$are, respectively, the neutral and the charged Goldstone bosons. $X$ and $A$ are the new neutral physical scalars ${ }^{1}$ and $H^{ \pm}$is the charged physical scalar. While both $X$ and $A$ are possible DM candidates, the physics is the same through the interchange $\left(\lambda_{5}, X\right) \leftrightarrow\left(-\lambda_{5}, A\right)$. In these series of papers we take, for definiteness, $X$ as the DM

\footnotetext{
${ }^{1}$ Since these additional scalars do not couple to the fermions (of the SM), we can not assign them definite $C P$ numbers. By an abuse of language, we will, nonetheless, call $A$ the pseudoscalar.
}

candidate. Note that, in order to avoid any confusion between the neutral scalars of the model, we have labeled the fields differently than in our previous paper [10].

It is much instructive to revert to the description of the model through the physical parameters, especially when we will be seeking, as much as possible, an on shell renormalization of the model based on physical observables. In particular, the parameters of the potential can be translated to the physical masses of the scalars,

$$
\begin{gathered}
M_{h}^{2}=2 \lambda_{1} v^{2}, \\
M_{H^{ \pm}}^{2}=\mu_{2}^{2}+\lambda_{3} \frac{v^{2}}{2}, \\
M_{X}^{2}=\mu_{2}^{2}+\lambda_{L} \frac{v^{2}}{2}=M_{H^{ \pm}}^{2}+\left(\lambda_{4}+\lambda_{5}\right) \frac{v^{2}}{2}, \\
M_{A}^{2}=\mu_{2}^{2}+\lambda_{A} \frac{v^{2}}{2}=M_{H^{ \pm}}^{2}+\left(\lambda_{4}-\lambda_{5}\right) \frac{v^{2}}{2}=M_{X}^{2}-\lambda_{5} v^{2}, \\
\text { where } \lambda_{L / A}=\lambda_{3}+\lambda_{4} \pm \lambda_{5} .
\end{gathered}
$$

Unfortunately, the masses do not provide enough input to determine all the independent parameters of the potential. First of all, $\lambda_{2}$ is a parameter that describes the interaction solely within the dark sector $X, A, H^{ \pm}$. Therefore, at tree level, the SM particles are insensitive to this parameter even in their interaction with the dark sector particles $X, A, H^{ \pm}$particles. Nonetheless, as we will see, one-loop observables will depend on this parameter that describes scattering/rescattering in the DM sector. Second, to fully define an observable, we still need an extra parameter, $\mu_{2}, \lambda_{3}$, or alternatively, $\lambda_{L}$. The latter seems to be the most appropriate combination since it has a direct physical interpretation. Indeed, the coupling of the SM Higgs boson, $h$, to a pair of DM is (at the amplitude level) given by

$$
\mathcal{A}_{h X X}^{0}=-\lambda_{L} v .
$$

The superscript ${ }^{0}$ relates to the tree-level definition. In lieu of the mass term, $\mu_{2}^{2}$, we take $\lambda_{L}$ as an input parameter. The parameters of the potential in Eq. (2.2) can then be reconstructed from the input parameters $\left(M_{h}, M_{X}, M_{A}\right.$, $\left.M_{H}^{ \pm}, \lambda_{L}\right)$ as

$$
\begin{aligned}
& \lambda_{1}=\frac{M_{h}^{2}}{2 v^{2}}, \quad\left(\lambda_{1} \sim+0.129\right), \\
& \lambda_{5}=\frac{M_{X}^{2}-M_{A}^{2}}{v^{2}} \\
& \lambda_{4}=\lambda_{5}+2 \frac{M_{A}^{2}-M_{H^{ \pm}}^{2}}{v^{2}} \\
& \lambda_{3}=\lambda_{L}-\lambda_{4}-\lambda_{5}
\end{aligned}
$$




$$
\left(\mu_{2}^{2}=M_{X}^{2}-\lambda_{L} \frac{v^{2}}{2} \rightarrow \lambda_{L}=\frac{2\left(M_{X}^{2}-\mu_{2}^{2}\right)}{v^{2}}\right) .
$$

$\mu_{2}$ is a redundant parameter. As mentioned earlier, $\lambda_{2}$ is a parameter, which, at tree level is not accessible since it describes the interactions solely within the dark sector. To define any benchmark and in view of the OS scheme for the renormalization of the IDM that we advocate, apart from the SM parameters, the input (physical) parameters we choose are

$$
M_{X}, M_{A}, M_{H^{ \pm}}, \lambda_{L},\left(\lambda_{2}\right) .
$$

It is instructive to make the following comments which will prove helpful later. When the DM candidate, $X$, is almost degenerate in mass with $A$, coannihilation may be important. This scenario requires $\lambda_{5} \sim 0$. In another limit, $M_{H^{ \pm}}=M_{A}$, the electroweak custodial symmetry parameter $T$ vanishes at one loop, even when $M_{A}, M_{H^{ \pm}}$are large. In this limit, with $M_{A, H^{ \pm}}>M_{X}, \lambda_{4}=\lambda_{5}=-\lambda<0$ (with $\lambda>0$ ). As we will see later, direct detection will impose that $\lambda_{L} \ll 1$, which means that $\lambda_{3} \sim 2 \lambda$ when the custodial symmetry is imposed.

\section{RENORMALIZATION OF THE MODEL}

\section{A. Automation of the calculation}

New model files for the $\mathrm{IDM}^{2}$ allowing different schemes for the renormalization of the model, to which we turn shortly, have been added to Sloops [2-6,8-10, 113,114]. SloopS, our automated code for the calculation of tree-level and one-loop observables relies on the bundle of packages based on FeynArts [115], FormCalc [116], and LoopTools [117], that we refer to as FFL for short. A few improvements to LoopTools [2] have been made over the years. A key component is the generation of the model file (with counterterms and renormalization conditions) made possible with LanHEP $[118,119]$ judiciously interfaced with the bundle FFL. We have exploited this approach successfully for the SM, the full renormalization of all sectors of the MSSM $[3,4,8]$, the next-to-minimal supersymmetric standard model (NMSSM) [114], and a singlet extension of the SM [9]. The code has been thoroughly checked and allows many tests on the correctness of the results, including tests on the ultraviolet finiteness (for loop calculations) and very importantly, on gauge parameter independence of the results both at tree level and at loop level; see III C below. The code is optimized also for DM

\footnotetext{
${ }^{2}$ The model files can be downloaded from the wikipage https:// lapth.cnrs.fr/projects/PrecisionCalculations/. They are provided for those who would like to conduct further precision analyses of the IDM in accord with the renormalization we explicit in this section. The present subsection explains which other codes are needed to run SloopS. Section VI will give the different steps to arrive at the derivation of the relic density.
}

annihilation cross section such that it inputs directly the result for $\sigma v$, where $\sigma$ is the annihilation cross section and $v$ is the relative velocity of the annihilating particles. This avoids potential instabilities when specializing to $v \rightarrow 0$ had the output been $\sigma$.

\section{B. The SM parameters}

The SM part of the IDM is renormalized exactly in the same way as what has become standard practice in oneloop calculations of electroweak observables [114]. This calls for an OS scheme whereby the fermion masses as well as the mass of the $W, M_{W}=80.449 \mathrm{GeV}$, the $Z, M_{Z}=$ $91.187 \mathrm{GeV}$, and the Higgs boson, $M_{h}=125 \mathrm{GeV}$, are taken as input physical masses. For the SM, the tadpole, $T_{\text {ad }}=v\left(\mu_{1}^{2}-\lambda_{1} v^{2}\right)$ (at tree level) is required to vanish at all orders. The electric charge is defined in the Thomson limit; see [3]. The light quark $(u, d, s, c)$ masses, $m_{u}=m_{d}=$ $66 \mathrm{MeV}, m_{s}=150 \mathrm{MeV}, m_{c}=1.6 \mathrm{GeV}$, are taken as effective quark masses that reproduce the SM value of $\alpha^{-1}\left(M_{Z}^{2}\right) \sim 128.907$. Use of the latter effective coupling can, in many instances, amount to about $13 \%$ correction compared to the use of $\alpha=\alpha(0)=1 / 137.036$. Since it is $\alpha\left(M_{Z}^{2}\right)$ that is used as the default value ${ }^{3}$ of the effective coupling in the tree-level evaluation of micrOMEGAs [107, 120-122], we will investigate whether implementing the numerical value $\alpha\left(M_{Z}^{2}\right)$ instead of $\alpha$ in tree-level cross sections can account for a significant part or the full oneloop correction. For a portion of the allowed parameter space of the IDM, the relic density is driven almost entirely through the SM Higgs resonance for which $h \rightarrow b \bar{b}$ is dominant. We will therefore take an effective $b$-quark mass that (at tree level) reproduces very well the dominant partial width to $b$ s and the total SM Higgs width. In fact, for such a scenario, we adapt the renormalization of the Higgs mass to include its width as well. We give more details [110] on this subtle but very important issue when we describe the processes for the DM annihilation that we will be dealing with. The top mass is taken as $m_{t}=174.3 \mathrm{GeV}$.

\section{Gauge fixing}

We take a nonlinear gauge fixing term $[2,3,109]$ that still preserves the $Z_{2}$ symmetry. We take these gauge fixing terms to be renormalized. In particular, the gauge functions involve the physical fields. Although this will not make all Green's functions finite, it is enough to make all $S$-matrix elements finite. With $A_{\mu}$, the photon field, we write the gauge fixing as

\footnotetext{
${ }^{3} \mathrm{We}$ stress this point in case users of micrOMEGAs attempt to compare the tree-level results we quote in this series of papers with the output of their runs with the default SM values of the input parameters in micrOMEGAs. We can of course run micrOMEGAs with $\alpha(0)$.
} 


$$
\mathcal{L}^{G F}=-\frac{1}{\xi_{W}} F^{+} F^{-}-\frac{1}{2 \xi_{Z}}\left|F^{Z}\right|^{2}-\frac{1}{2 \xi_{\gamma}}\left|F^{A_{\mu}}\right|^{2},
$$

where

$$
\begin{aligned}
F^{+}= & \left(\partial_{\mu}-i e \tilde{\alpha} \gamma_{\mu}-i e \frac{c_{W}}{s_{W}} \tilde{\beta} Z_{\mu}\right) W^{\mu+} \\
& +i \xi_{W} \frac{e}{2 s_{W}}\left(v+\tilde{\delta} h+i \tilde{\kappa} G^{0}\right) G^{+}, \\
F^{Z}= & \partial_{\mu} Z^{\mu}+\xi_{Z} \frac{e}{s_{2 W}}(v+\tilde{\epsilon} h) G^{0}, \\
F^{A_{\mu}}= & \partial_{\mu} A^{\mu} .
\end{aligned}
$$

The ghost Lagrangian $\mathcal{L}^{G h}$ is derived through the use of the BRST transformation, see [109], which leads to an easy implementation in the automated code Sloops. We specialize to the case $\xi_{W}=\xi_{Z}=\xi_{\gamma}=1$ in order that the gauge propagators retain the simple form of the usual Feynman gauge, but the nonlinear gauge furnishes enough parameters $(\tilde{\alpha}, \ldots \tilde{\epsilon})$ on which to carry out the gauge parameter dependence of the amplitude for further checks on the correctness of the calculation beside the ultraviolet finiteness tests. We perform gauge parameter independence checks on all cross sections and decays we calculate with Sloops in this series of papers.

\section{On shell renormalization for the masses of the IDM scalars}

The renormalization of the IDM is technically quite straightforward since the new scalars, $A, X$, and $H^{ \pm}$, do not mix, nor do they mix $(1 \rightarrow 1$ transition) with those of the $\mathrm{SM}$, including the Goldstones, because of the $Z_{2}$ symmetry. Shifts on the parameters of the potential, which get translated into counterterms, $\delta \lambda_{i}$, for the $\lambda_{i}$ (and $\mu_{2}$ ) as well as shifts on the fields through wave function renormalization constants $(\delta Z)$, are introduced. In the OS scheme, the masses of the physical scalars, $\phi\left(\phi=X, A, H^{ \pm}\right)$, are defined from the pole position of the one-loop corresponding self-energy $\Sigma_{\phi \phi}\left(k^{2}\right)$, where $k$ is the momentum carried by $\phi$. We also require that the residue at the pole of these particles be properly normalized to unity. The conditions on the counterterms are then

$$
\begin{gathered}
\delta M_{\phi}^{2}=\operatorname{Re} \Sigma_{\phi \phi}\left(M_{\phi}^{2}\right), \\
\delta Z_{\phi}=-\left.\operatorname{Re} \frac{\partial \Sigma_{\phi \phi}\left(k^{2}\right)}{\partial k^{2}}\right|_{k^{2}=M_{\phi}^{2}} .
\end{gathered}
$$

\section{E. $\lambda_{L}: \overline{\mathrm{MS}}$ and $\mathrm{OS}$}

We use the full $h \rightarrow X X$ amplitude to define $\lambda_{L}$. At tree level, the amplitude is given by Eq. (2.10). It serves to generate (and define) the counterterms for the one-loop amplitude. The latter also includes the contributions of the one-loop diagrams such that the full renormalized one-loop amplitude is momentum dependent. The full one-loop renormalized amplitude (when the threshold is open) for $h\left(Q^{2}\right) \rightarrow X\left(p_{1}^{2}\right) X\left(p_{2}^{2}\right)$ of the SM Higgs, $h$, with momentum $Q$ to a pair of the DM $X$ with momenta $p_{1}$, and $p_{2}$ writes as

$$
\begin{aligned}
\mathcal{A}_{h X X}^{\mathrm{ren}}\left(Q^{2}, p_{1}^{2}, p_{2}^{2}\right)= & -\left(v \delta \lambda_{L}+\lambda_{L} \delta v+\lambda_{L} v\left(\frac{1}{2} \delta Z_{h}+\delta Z_{X}\right)\right) \\
& +\mathcal{A}_{h X X}^{\mathrm{lPI}}\left(Q^{2}, p_{1}^{2}, p_{2}^{2}\right),
\end{aligned}
$$

where $\mathcal{A}_{h X X}^{1 \mathrm{PI}}\left(Q^{2}, p_{1}^{2}, p_{2}^{2}\right)$ is the full one-loop one-particle irreducible vertex. $\delta \lambda_{L}$ is the counterterm for $\lambda_{L}$ for which we are seeking a renormalization condition, $\delta Z_{h}$ is the SM wave function renormalization and $\delta Z_{X}$ is the wave function renormalization for the DM particle, $X . \delta v$ is the counterterm for $v$ [defined through $e, M_{Z}, M_{W}$ in Eq. (2.4)]. When the threshold for the ziggs decay to $X X$ is open, we set $Q^{2}=M_{h}^{2}$ and $p_{1}^{2}=p_{2}^{2}=M_{X}^{2}$ and require that the full one-loop correction to this partial width is zero, defining a gauge invariant OS counterterm for $\lambda_{L}$ as

$$
\begin{aligned}
\delta^{\mathrm{OS}} \lambda_{L}= & \frac{\mathcal{A}_{h X X}^{1 \mathrm{PI}}\left(Q^{2}=M_{h}^{2}, M_{X}^{2}, M_{X}^{2}\right)}{v} \\
& -\lambda_{L}\left(\frac{\delta v}{v}+\frac{1}{2} \delta Z_{h}+\delta Z_{X}\right) \text { for } M_{h}>2 M_{X} .
\end{aligned}
$$

Another gauge invariant but scale dependent scheme valid even when the decay threshold is closed, is to use a $\overline{\mathrm{MS}}$ definition, where only the (mass independent term) ultraviolet divergent part is kept.

$$
\begin{aligned}
\delta^{\overline{\mathrm{MS}}} \lambda_{L} & =\left(\frac{\mathcal{A}_{h X X}^{1 \mathrm{PI}}\left(Q^{2}, M_{X}^{2}, M_{X}^{2}\right)}{v}-\lambda_{L}\left(\frac{\delta v}{v}+\frac{1}{2} \delta Z_{h}+\delta Z_{H}\right)\right)_{\infty} \\
& \text { for any } Q^{2} .
\end{aligned}
$$

In our code, $\delta^{\mathrm{OS}} \lambda_{L}$ in Eq. (3.6) and $\delta^{\overline{\mathrm{MS}}} \lambda_{L}$ in Eq. (3.7) are extracted exactly by evaluating the amplitude $h \rightarrow X X$ and constructing the above equations.

In our previous paper, dealing with the heavy DM scenario [10], we did mention the possibility of using as input another, OS observable to define the $\lambda_{L}$ counterterm. Formally, the scattering of a quark, $q$, and the DM, $X$, $X q \rightarrow X q$ involves, as a subset, the exchange of the SM Higgs boson. The matrix element of such a process is therefore sensitive to $\lambda_{L}$. This is exactly what is at play in direct detection, where the exchange occurs at very small $Q^{2} \sim 0 \mathrm{GeV}^{2}$. However, this is impractical. At the level of the nuclear matrix elements that enter the detection rate, many other contributions need to be evaluated (gluonic contributions, ...), and large uncertainties have to be taken 
into account such that the extraction of the effective coupling $h X X$ will not be fit for an input for a precision calculation, let alone that the effective coupling refers to a scale $Q^{2} \sim 0$, which is far removed from typical scales where cross sections for DM annihilation take place. In Ref. [46], a very interesting proposal for defining the $\lambda_{L}$ counterterm was made. In essence, it is suggested that, for $M_{X}=M_{h} / 2$, one could use the measured value of the relic density itself. In this extremely narrow range of the DM mass, the relic density is extremely sensitive to $\lambda_{L}$. Assuming nonresonant contributions to be negligible, this amounts to isolating the $h \rightarrow X X$ decay amplitude. This in turn provides a way of calculating radiative correction to direct detection. ${ }^{4}$ Away from $M_{X}=M_{h} / 2$, a scale dependence is introduced but more importantly, there is an issue with the nonresonant contributions in the relic density calculation, which were not estimated. We prefer therefore the use of the $h \rightarrow X X$ amplitude, which isolates the $\lambda_{L}$ parameter unambiguously.

\section{F. The $\beta$ constant for $\boldsymbol{\beta}_{\lambda_{L}}$}

The coefficient of the ultraviolet divergent part is nothing but the one-loop $\beta$ constant for $\lambda_{L}$,

$$
\delta^{\overline{\mathrm{MS}}} \lambda_{L}=\frac{1}{32 \pi^{2}} \tilde{\beta}_{\lambda_{L}} C_{\mathrm{UV}}, \quad C_{\mathrm{UV}}=-\frac{2}{\varepsilon}-1+\gamma_{E}-\ln (4 \pi),
$$

where $\varepsilon=4-d$ with $d$ being the number of dimensions in dimensional regularization and $\gamma_{E}$ is the Euler-Mascheroni constant. Or, keeping $\mu_{\mathrm{dim}}$, the scale introduced by dimensional regularization [which goes hand in hand with the $\ln (4 \pi)$ term as $\ln \left(4 \pi \mu_{\text {dim }}^{2}\right)$ ], the scale, $Q^{2}$, dependence through the parameter $\lambda_{L}$ also writes as

$$
32 \pi^{2} \frac{\partial \lambda_{L}}{\partial \ln \left(Q^{2}\right)}=-32 \pi^{2} \frac{\partial \lambda_{L}}{\partial \ln \left(\mu_{\mathrm{dim}}^{2}\right)}=\tilde{\beta}_{\lambda_{L}}
$$

The analytical formulas for the $\beta$ constants of the IDM have been adapted from those of the general two Higgs doublet model (2HDM) [94,123] and can be found in [71,98]. For our purposes, we have reformulated them for $\lambda_{L}$, taking into account the scalar(s), the gauge (g) and the Yukawa (Y) contributions as

$$
\tilde{\beta}_{\lambda_{L}}=\tilde{\beta}_{\lambda_{L}}^{(g)}+\tilde{\beta}_{\lambda_{L}}^{(s)}+\tilde{\beta}_{\lambda_{L}}^{(Y)}
$$

with

\footnotetext{
${ }^{4} \mathrm{~A}$ variation on this proposal for the coannihilation region was recently discussed in Ref. [51].
}

$$
\begin{aligned}
\tilde{\beta}_{\lambda_{L}}^{(g)}= & -3 \lambda_{L}\left(3 g^{2}+g^{\prime 2}\right)+\frac{3}{4}\left(3 g^{4}+g^{4}+2 g^{2} g^{\prime 2}\right) \\
\tilde{\beta}_{\lambda_{L}}^{(s)}= & 4 \lambda_{L}\left(\lambda_{L}+3\left(\lambda_{1}+\lambda_{2}\right)\right)-4\left(\lambda_{1}+\lambda_{2}\right)\left(\lambda_{4}+2 \lambda_{5}\right) \\
& +2\left(\lambda_{4}^{2}+2 \lambda_{4} \lambda_{5}+3 \lambda_{5}^{2}\right) \\
\tilde{\beta}_{\lambda_{L}}^{(Y)}= & 4 \lambda_{L} \sum_{f=\text { all fermions }} N_{C}^{f} \frac{m_{f}^{2}}{v^{2}}
\end{aligned}
$$

Here, $m_{f}$ is the mass of the fermion, $f$, and $N_{c}^{f}$ is its corresponding color factor (3 for quarks and 1 for leptons). For small $\lambda_{L}$, the Yukawa contribution is tiny, and the largest contribution is from the top quark. We find excellent agreement (in fact perfect agreement with machine precision) between these analytical formulas and those extracted from our code according to Eqs. (3.7), (3.8). An important observation here is that Eq. (3.11) shows that even if $\lambda_{L}=0$, a one-loop induced $\lambda_{L}$ is generated. Also, while $\lambda_{2}$ is a parameter residing solely in the dark sector and is not involved at tree level in DM annihilation processes to SM particles, it makes its effect felt at one loop with the conclusion that a large scale variation due to $\lambda_{2}$ can be present apart from other contributions at one loop. In our code, we can freely vary $\mu_{\text {dim }}^{2}$ to quantify the scheme dependence in an $\overline{\mathrm{MS}}$ scheme. Obviously, in a fully OS scheme, the check on the UV finiteness of the result means that there is no $\mu_{\mathrm{dim}}^{2}$ dependence.

\section{G. Scale dependence of the one-loop corrected $h \rightarrow X X$ in the $\overline{\mathrm{MS}}$}

At tree level, the partial width for Higgs decay to a pair of DM, $X$, is expressed as

$\Gamma_{h \rightarrow X X}=\frac{\lambda_{L}^{2} v^{2}}{32 \pi M_{h}} \sqrt{1-\frac{4 M_{X}^{2}}{M_{h}^{2}}}, \quad\left(M_{h}>2 M_{X}\right)$,

showing that the parametric dependence on $\lambda_{L}$, a quadratic dependence, of this observable is algebraically straightforward. This is the reason that this observable is chosen as an input to define $\lambda_{L}$. Naturally, in the OS scheme, this observable receives no correction. Nonetheless, as we will see later, based on present experimental constraints, which means that the Higgs partial decay rate to DM is way too small to be measured, let alone be measured with good precision, one may be forced to rely on an $\overline{\mathrm{MS}}$ prescription. When $h \rightarrow X X$ is closed, the use of the OS scheme is not possible, and in this case, we have to study the scale uncertainty.

The purpose of this short interlude is to investigate which scale choice may be considered the best, best in the sense of minimizing the one-loop correction. In fact, $\Gamma_{h \rightarrow X X}$ provides a good example. One can study this observable at one loop in the $\overline{\mathrm{MS}}$ and quantify how it deviates from the tree-level result which is, by construction, the result of the OS scheme. Knowing the $\lambda_{L}$ dependence of an observable at tree level 
and having at our disposal the $\beta_{\lambda_{L}}$ of the model, the scale dependence can be derived analytically allowing to compare the difference between two choices of scale, $\mu_{1}$ and $\mu_{2}$.

The $\lambda_{L}$ dependence of $\Gamma_{h \rightarrow X X}$ is trivial. Indeed, from Eq. (3.12), we have

$$
\frac{\delta \Gamma_{h \rightarrow X X}}{\Gamma_{h \rightarrow X X}}=2 \frac{\delta \lambda_{L}}{\lambda_{L}}
$$

which through Eq. (3.9) allows us to relate the one-loop correction at scale $\mu_{2}$ to that at scale $\mu_{1}$ as

$$
\frac{\delta \Gamma_{h \rightarrow X X}\left(\bar{\mu}_{2}\right)-\delta \Gamma_{h \rightarrow X X}\left(\bar{\mu}_{1}\right)}{\Gamma_{h \rightarrow X X}^{\text {(tree) }}}=-\frac{1}{8 \pi^{2}} \frac{\tilde{\beta}_{\lambda_{L}}}{\lambda_{L}} \ln \left(\bar{\mu}_{2} / \bar{\mu}_{1}\right) .
$$

As expected, an important lesson to always keep in mind is that large $\tilde{\beta}_{\lambda_{L}}$ values induce large scale variations. For the IDM, $\beta_{\lambda_{L}}$ depends also on $\lambda_{2}$ which can contribute significantly. These considerations are crucial. However, they do not point to the most optimal choice of $\mu$. The most optimal choice of $\mu$ is the one that minimizes the full oneloop correction. The latter will involve all scales of the problem. Therefore, to investigate the issue of the optimal scale, we need to consider a few examples calculating the full one-loop correction.

For the purpose of this exercise, we take a value of $\lambda_{L}$ which is not too small in order not to induce unnaturally large relative corrections. At this stage, we do not impose experimental constraints on the parameter space. This is studied in great detail, later. Our aim here is to see if there is a trend for an optimal choice of the scale that we could then advocate for other processes driving the relic density. We consider three models that differ in the masses of $A$ and $H^{ \pm}$. With $M_{h}=125 \mathrm{GeV}$, we keep $M_{X}=57 \mathrm{GeV}$ for these three models. These choices generate different hierarchies for the scale of the problem. While $\lambda_{2}$ is not needed to calculate the tree-level decay, the value of $\lambda_{2}$ is required at one loop. We consider three values of $\lambda_{2}$ for each point to gauge the $\lambda_{2}$ dependence, and we test various values of the scale, $\mu$, in relation with the scales that are involved in the observable at one loop. It is important to observe that the masses of the IDM act at two levels in the one-loop result. Reinterpreted in terms of $\lambda_{i=3,4,5,(L)}$, they contribute directly to the coefficient $\tilde{\beta}_{\lambda_{L}}$ as $\lambda_{2}$ does, but unlike $\lambda_{2}$, the masses are involved in the arguments of the various oneloop scalar functions.

Our results of the numerical full one-loop corrections are shown in Table I. First of all, Eq. (3.14) is in perfect agreement with the numbers given in Table I, where we compare, for the same point, the correction between two scales. This is another evidence of the correctness of the implementation of the model and the numerical computation.
TABLE I. One-loop corrections, $\delta \Gamma_{h \rightarrow X X}$, to the partial decay width of the SM Higgs boson $\left(M_{h}=125 \mathrm{GeV}\right)$ to pairs of DM, $X$, with $M_{X}=57 \mathrm{GeV}$ (all values for the widths are given in $\mathrm{MeV}$ ) in the $\overline{\mathrm{MS}}$ scheme for different masses of $M_{H^{ \pm}}$and $M_{A}$ and $\lambda_{2}$, the coupling within the dark sector. The tree-level width of this observable is $5.106 \mathrm{MeV}$. All masses (and scales) are in GeV. For each set of parameters defining the IDM model, we give the reconstructed $\lambda_{i}$ parameters as well as the one loop $\tilde{\beta}$. The parameters of the model are only illustrative and do not necessarily pass the experimental constraints on the IDM. Entries in bold represent corrections that represent more than $75 \%$ of the tree-level value, while those that are also underlined lead to a total

\begin{tabular}{|c|c|c|c|c|}
\hline (Model) $\lambda_{L} ; M_{A}, M_{H^{ \pm}}$ & $\mu_{\mathrm{dim}}$ & $\lambda_{2}=0$. & $\lambda_{2}=1$ & $\lambda_{2}=2$ \\
\hline (A) $0.05 ; 106,106$ & $M_{h} / 2$ & 0.90 & 1.48 & 2.05 \\
\hline $\begin{array}{l}\lambda_{4}=\lambda_{5}=-0.127 \\
\quad \lambda_{3}=0.305\end{array}$ & $M_{h}$ & $-1.910^{-2}$ & -1.36 & -2.69 \\
\hline \multirow[t]{5}{*}{$\left(\tilde{\beta}_{\lambda_{L}}=1.03+2.13 \lambda_{2}\right)$} & $2 M_{h}$ & -0.94 & -4.18 & -7.44 \\
\hline & $M_{X}$ & 1.02 & 1.85 & 2.68 \\
\hline & $2 M_{X}$ & 0.10 & -0.98 & -2.06 \\
\hline & 200 & -0.64 & -3.28 & $-\mathbf{5 . 9 0}$ \\
\hline & $M_{A}=M_{H^{ \pm}}$ & 0.2 & -0.68 & -1.56 \\
\hline (B) $0.05 ; 138,138$ & $M_{h} / 2$ & 1.73 & 4.47 & 7.20 \\
\hline $\begin{array}{c}\lambda_{4}=\lambda_{5}=-0.252 \\
\lambda_{3}=0.555\end{array}$ & $M_{h}$ & 0.13 & -0.38 & -0.90 \\
\hline \multirow[t]{5}{*}{$\left(\tilde{\beta}_{\lambda_{L}}=1.78+3.63 \lambda_{2}\right)$} & $2 M_{h}$ & -1.46 & -5.23 & -9.01 \\
\hline & $M_{X}$ & 1.95 & 5.11 & $\underline{8.28}$ \\
\hline & $2 M_{X}$ & 0.35 & 0.26 & $\overline{0.17}$ \\
\hline & 200 & -0.95 & -3.67 & -6.40 \\
\hline & $M_{A}=M_{H^{ \pm}}$ & -0.10 & -1.07 & -2.06 \\
\hline (C) $0.05 ; 170,200$ & $M_{h} / 2$ & 6.51 & 15.5 & 24.4 \\
\hline $\begin{array}{c}\lambda_{4}=\lambda_{5}=-0.765 \\
\lambda_{3}=1.225\end{array}$ & $M_{h}$ & 2.15 & 4.87 & 7.60 \\
\hline \multirow[t]{5}{*}{$\left(\tilde{\beta}_{\lambda_{L}}=4.86+6.94 \lambda_{2}\right)$} & $2 M_{h}$ & -2.21 & -5.71 & -9.21 \\
\hline & $M_{X}$ & 7.08 & 16.9 & 26.6 \\
\hline & $2 M_{X}$ & 2.72 & 6.28 & 9.83 \\
\hline & $M_{H^{ \pm}}$ & -0.81 & -2.30 & -3.80 \\
\hline & $M_{A}$ & 0.22 & 0.18 & 0.15 \\
\hline
\end{tabular}
width that turns negative.

The relevant scales of the problem are all the invariants that are involved in the loop functions that enter the radiative corrections. They include both the external kinematical variables but also the internal masses called in the loop calculations. The $\mu_{\mathrm{dim}}$ dependence is tracked as $\log \left(Q_{\Delta}^{2} / \mu_{\text {dim }}^{2}\right) \cdot Q_{\Delta}$ is an effective collective scale which is a combination of the scales involved in the (various) loops entering the loop calculation. As argued at some length in Ref. [114], if one of these scales is (much) larger than the others, it should approximate $Q_{\Delta}$.

One would expect that the typical scale for this decay is $M_{h}$. Observe that in our case, $M_{h} \sim 2 M_{X}$. Table I shows that $\mu_{\mathrm{dim}}=M_{h}, 2 M_{X}$ is a good choice but only if $M_{A}<M_{h}, 2 M_{X}$. In particular, for the case (C) of Table I where $M_{A}$ is much larger that $M_{h}$ and $2 M_{X}, M_{A}$ is by far the best choice for all values of $\lambda_{2}$ confirming the general 
observation made in [114] in the totally different context of the NMSSM, where it was found that the largest scale minimizes the correction. We must also keep in mind that when $M_{A}$ is much larger than $M_{X}, \beta_{\lambda_{L}}$ is larger (since $\lambda_{3,4,5}$ are larger). It is therefore more important to choose $M_{A}$ as the optimal $\mu_{\mathrm{dim}}$. As a rule of thumb, we advocate the optimal scale to be $\mu=\max \left(M_{h}, M_{A}\right), M_{h}$ is the typical (kinematical) scale of the process, while $M_{A}$ is the typical internal scale. We find that it fares better than $M_{H^{ \pm}}$. Scales that are much smaller or much larger than the optimal scale, for instance, $M_{X}, M_{h} / 2,2 M_{h}$, lead to large corrections. On many instances these corrections are so large that the calculations are not reliable.

We check this conjecture about the optimal scale also in the study of the annihilation cross section of DM, $X X \rightarrow \mathrm{SM}$. Considering the rather small velocities taking part in the annihilation cross sections, $2 M_{X}$ will again represent the typical kinematical scale to be compared again with the internal mass, $M_{A}$.

\section{PRESENT CONSTRAINTS ON THE PARAMETER SPACE OF THE IDM}

We quantify the effect of the one-loop corrections on the annihilation cross sections in the IDM. We perform these calculations on some benchmark points of the IDM. The benchmark points need to pass several constraints. While searching for benchmark points that satisfy all theoretical and experimental constraints, we perform a numerical scan, where all the constraints that we discuss in this section are implemented numerically without any approximation, by running different codes and routines. Nonetheless, it is instructive to extract the salient features through approximate analytical formulas, and some judicious considerations as this helps carry out a more efficient scan. Thorough investigations of the constraints have been performed recently $[78,83] .{ }^{5}$ While we agree with their findings, we update the constraints in view of some new experimental data on the direct detection of dark matter and searches/ analyses at the LHC. Our purpose is not to present new exclusion zones in the parameter space of the model but to make sure that the benchmark points we pick up for our studies at one loop, pass all the experimental (and theoretical constraints), and are representative of a larger set. As is known, the present precision on the relic density of DM is such that it reduces the parameter space of any model of DM, drastically. However, since all studies impose this constraint based on tree-level calculations of the annihilation cross sections, we use the tree-level based relic density constraint only as a guide and see how the prediction transforms when a full one-loop calculation is implemented and how the theoretical uncertainties should be taken into account. Many studies have allowed benchmarks where the

\footnotetext{
${ }^{5}$ See also $[23,46,58-87,124]$.
}

predicted (tree-level) relic density leads to underabundance but not (obviously) overabundance. Nevertheless, a prediction of an overabundance with a tree-level calculation may turn into a viable model with a more precise calculation. First of all, let us repeat again that we are taking $X$ as the DM candidate. $X$ is neutral and is taken to be the lightest of the three additional scalars that the IDM provides. From (2.11), this means that

$$
\lambda_{5}<0
$$

Moreover, we take $M_{A} \leq M_{H^{ \pm}},{ }^{6}$ in which case,

$$
\lambda_{4} \leq \lambda_{5}<0 .
$$

The equality $\left(\lambda_{4}=\lambda_{5}\right)$ automatically evades indirect electroweak precision measurements by preserving custodial symmetry. Remember also that the existence of the SM Higgs boson means that

$$
\lambda_{1}>0 .
$$

Usually while studying the viable parameter space of the IDM, the discussion starts with the restriction from the stability and the perturbativity of the potential. We discuss all these constraints, but we prefer to start with a constraint that has in the last years become quite stringent. It also delimits a specific coupling and not a combination of a large number of parameters. It concerns the parameter $\lambda_{L}$ and its connection to the DM direct detection. $\lambda_{L}$ is a key input in our OS renormalization scheme.

\section{A. Dark matter direct detection}

The direct detection of dark matter sets a very strong constraint on $\lambda_{L} / M_{X}$ for the IDM. In the rather narrow (light) mass range, direct detection proceeds through the SM Higgs exchange with a cross section given by

$$
\sigma_{\mathrm{SI}}^{\mathrm{h}} \sim\left(\frac{\lambda_{L} \times 10^{3}}{M_{X} / 100 \mathrm{GeV}}\right)^{2} 8.53 \times 10^{-49} \mathrm{~cm}^{2} .
$$

Assuming that $X$ accounts for all of the DM density on Earth and provides the correct measured relic density as we are assuming, the latest xenon1T limit [125] for a DM in the range $50<M_{X}<100 \mathrm{GeV}$ translates into

\footnotetext{
${ }^{6}$ This choice does not allow for coannihilation $X H^{ \pm} \rightarrow f \bar{f}^{\prime}$ when $X A \rightarrow f \bar{f}$ coannihilation is not present.

${ }^{7}$ Again, here our analysis for direct detection is at tree level. There may be important radiative corrections, but these can be incorporated only within a coherent renormalization scheme and a global analysis of different observables within the same scheme. Please refer to our discussion at the end of paragraph 3.5. In any case, we do not expect an order of magnitude change.
} 


$$
\sigma_{\mathrm{SI}}^{\mathrm{Xenon} 1 \mathrm{~T}}<12 \times 10^{-47} \times\left(M_{X} / 100 \mathrm{GeV}\right) \mathrm{cm}^{2} .
$$

Applied to our case, we have

$$
\begin{aligned}
\left|\lambda_{L}\right| \times 10^{3} & <12 \times\left(M_{X} / 100 \mathrm{GeV}\right)^{3 / 2} \\
\rightarrow\left|\lambda_{L}\right| & <\left|\lambda_{L}^{\max }\right|=7 \times 10^{-3}, \quad \text { for } M_{X}=70 \mathrm{GeV}, \\
\rightarrow\left|\lambda_{L}\right| & <5 \times 10^{-3} \quad \text { for } M_{X}=57 \mathrm{GeV} .
\end{aligned}
$$

This invisible branching fraction is far too small compared to the present limit from the LHC fits [126-128]. Indeed, from Eq. (3.12),

$$
\begin{aligned}
& \operatorname{Br}(h \rightarrow X X) \\
& =\left(1.183\left(\lambda_{L} \times 10^{3}\right)^{2} \sqrt{1-0.64\left(M_{X}(\mathrm{GeV}) / 50\right)^{2}}\right) \times 10^{-3}
\end{aligned}
$$

$$
=2.8 \times 10^{-3} \quad \text { for } M_{X}=57 \mathrm{GeV}, \quad \lambda_{L}=2.4 \times 10^{-3} .
$$

Considering the very tiny value of the invisible width, we take the total Higgs width to be the SM value of the total width from $[129,130]$

$$
\Gamma_{h}^{\mathrm{SM}}=4.07 \pm 0.16 \mathrm{MeV} .
$$

If the IDM only provides a fraction of the total relic density of DM, the direct detection cross section needs to be rescaled. With the density of the IDM written as $\Omega_{\mathrm{X}}$ and that extracted from the Planck measurements as $\Omega_{\mathrm{DM}}^{\text {Planck }}$, the limits above are transformed into

$$
\lambda_{L}<\lambda_{L}^{\max } \sqrt{\frac{\Omega_{\mathrm{DM}}^{\text {Planck }}}{\Omega_{\mathrm{X}}}},
$$

where $\lambda_{L}^{\max }$ is derived from Eq. (4.6). We take the view that if the IDM is to be considered as a model for DM, it should provide at least $50 \%$ of the total DM. This requirement does not significantly change the limit on $\lambda_{L}$ from direct detection constraints (a modest factor of $\sqrt{2}$ is possible), which is restricted to be rather small, $\lambda_{L}<0.01$.

\section{B. Electroweak precision observables (EWPO)}

The custodial $S U(2)$ symmetry breaking parameter, $T$ [131], restricts mass splitting between the scalars of the IDM. In the limit $M_{X} \ll M_{A}, M_{H}$, we require $M_{A} \sim M_{H}$. Indeed,

$\Delta T \simeq \frac{1}{24 \pi^{2} \alpha v^{2}} M_{A}\left(M_{H^{ \pm}}-M_{A}\right) \sim 0.05 \frac{M_{A}}{500 \mathrm{GeV}} \frac{\Delta M}{10 \mathrm{GeV}}$, is combined with the $S$ [131] parameter, which gives the weaker constraint,

$$
\Delta S \simeq-\frac{5}{72 \pi} .
$$

The full expressions for the $S, T$ contribution in the IDM can be found in Ref. [53].

In the code, we impose [132]

$S=0.06 \pm 0.09$,

$T=0.10 \pm 0.08$, with a correlation coefficient of +0.89 .

\section{Stability of the potential}

As with $\lambda_{1}>0$, we need to have $\lambda_{2}>0$. Equations (4.1) and (4.2) are sufficient to guarantee the vacuum to be neutral $\left(\lambda_{4}-\left|\lambda_{5}\right|<0\right)$. The other constraints on the potential (see, for example, [83]) are easily satisfied if one takes into account that direct detection requires a very small $\lambda_{L}$. The conditions expressed as a function of $\lambda_{L}$ require, for instance,

$$
2 \sqrt{\lambda_{1} \lambda_{2}}+\lambda_{L}>0
$$

which is satisfied if we take $\lambda_{L}>0$. In our case, the condition $2 \sqrt{\lambda_{1} \lambda_{2}}+\lambda_{3}=2 \sqrt{\lambda_{1} \lambda_{2}}+\lambda_{L}-\lambda_{4}-\lambda_{5}>0$ would be redundant.

Moreover, with very small $\lambda_{L}$ and not vanishingly small $\lambda_{2}$, the parameter, $R=\frac{\lambda_{L}}{2 \sqrt{\lambda_{1} \lambda_{2}}}$, satisfies $R \ll 1$, and we have that the minimum of the potential, which is the trivial one with the inert minimum being the deepest [83]. Requiring

$$
\lambda_{2}>0.01
$$

satisfies these conditions. An upper limit on $\lambda_{2}\left(\lambda_{2}\right.$ enters at one-loop order in the relic density calculation) is derived by considering unitarity to which we now turn to.

\section{Tree-level unitarity}

Unitarity constraints (that are found to be stronger than the perturbativity constraints) are also imposed; see [103]. These have been studied by considering the eigenvalues of the full set of all scattering processes involving all the scalars in the theory. They allow us to set an upper limit on the masses of the scalars. For $M_{H^{ \pm}} \gg M_{X}$, these constraints simplify if we impose the $T$ parameter constraints, $M_{A}=$ $M_{H^{ \pm}}\left(\right.$or $\left.\lambda_{4}=\lambda_{5}=-\lambda\right)$. They can be decomposed into subsets. The $\lambda_{1}, \lambda_{2}$ independent limits translate into 


$$
M_{A} \leq \sqrt{\frac{8 \pi}{3}} v \sim 720 \mathrm{GeV}
$$

Stronger limits apply if we allow for non-negligible values of $\lambda_{2}$. In the approximation, $\lambda_{4}=\lambda_{5}$ and with $\lambda_{L} \sim 0$, we have the additional constraint,

$$
\left(\lambda_{1}+\lambda_{2}\right)+\sqrt{\left(\lambda_{1}+\lambda_{2}\right)^{2}+\lambda^{2}}<\frac{8 \pi}{3},
$$

which, for small $\lambda_{2}$, gives the limit in Eq. (4.16). For $\lambda_{2}=2(1)$, values as high as $M_{A}=600(660) \mathrm{GeV}$ are within the perturbative tree-level regime. Tree-level unitarity allows much larger values of $\lambda_{2}$ than 2, especially for not too large values $M_{H^{ \pm}} \sim M_{A}$ as implied from Eq. (4.17). We have however restricted our analyses to $\lambda_{2}=0.01,1,2$.

\section{E. Collider limits}

\section{Direct searches at LEP}

The LEP constraints can be easily evaded if the masses of the inert scalars are above the threshold for LEPII production. In our benchmarks, this applies to $H^{+} H^{-}$pair production. For $X A$ associated production at LEPII, avoiding the constraints is possible either because the cross section is much reduced (close to threshold) and/or because the signature is such that these particles go undetected. Reinterpretation in terms of the IDM of LEPII data for searches of charginos [133] and neutralinos [61] has been done. These constraints can be summarized simply as $M_{A}, M_{H^{ \pm}}>110 \mathrm{GeV}$ independently of the $X$ mass (as long as $\left.M_{X}<M_{A, H^{ \pm}}\right)$. There is however a caveat when $\Delta M_{A X}=M_{A}-M_{X}<8 \mathrm{GeV}$ that leads to too soft leptons that invalidate the LEP searches. This recently discovered small region $[83,134]$ allows efficient $X A$ coannihilation into a fermion pair. We study this region and look at the impact of the loop corrections on the relic density in Ref. [111].

\section{Direct searches at the $\mathrm{LHC}$}

The searches (and limits on the parameter space) are based primarily on the Drell-Yan like associated production of the scalars. The signatures are classified according to the number, $\ell$, of charged leptons, missing energy and possibly jets,

$$
\begin{gathered}
H^{ \pm} X \rightarrow W^{ \pm} X X \rightarrow \ell^{ \pm}+\mathscr{E}_{T}, \quad \ell=1 \\
A X \rightarrow(Z) X X \rightarrow \ell^{+} \ell^{-}+\mathscr{E}_{T}, \quad \ell=2 \quad(\text { mono-Z) } \\
H^{ \pm} A \rightarrow\left(W^{ \pm}\right)(Z) X X \rightarrow \ell^{ \pm} \ell^{+} \ell^{-}+\mathbb{E}_{T}, \quad \ell=3 \\
H^{+} H^{-} \rightarrow\left(W^{+}\right)\left(W^{-}\right) X X \rightarrow \ell^{+} \ell^{-}+\mathbb{E}_{T}, \quad \ell=2,
\end{gathered}
$$

where the $Z, W^{ \pm}$may or may not be on shell. The monojet signature $(X X j, X A j)$ is relevant only when $\lambda_{L}$ is not too small $(X X j)$ or when $M_{A}-M_{X}$ is very small [83]. The contribution from vector boson fusion is negligible [83]. Higher values of $\ell=4,5$ can be envisaged depending on the mass difference between $M_{A}$ and $M_{H^{ \pm}}$giving more leptons through cascade decays [134].

The most studied $[59,135]$ scenario is the dilepton scenario in Eq. (4.19). Reinterpretation of the ATLAS and CMS $8 \mathrm{TeV}$ data concerning searches of charginos, neutralinos, and sleptons, to the case of the IDM was conducted in Ref. [79]. They are essentially based on the dilepton signature of the IDM, Eq. (4.19), with the important caveat that dileptons from the $Z$-boson decay are vetoed by the CMS/ATLAS cut $\left(\left|m_{\ell \ell}-M_{Z}\right|>10 \mathrm{GeV}\right)$. Combined with the LEP data, one learns $[78,79,83]$ that one should impose ${ }^{8}$

$$
M_{X}>45 \mathrm{GeV} .
$$

Simulations for all process (4.18)-(4.21) were studied for some benchmark points in [134], while a full simulation was performed for (4.19) in Ref. [83]. The caveat, $\left|m_{\ell \ell}-M_{Z}\right|>10 \mathrm{GeV}$, we alluded to earlier, has a crucial impact on the searches, existing and forthcoming. This cut is meant to reduce the large SM background in events containing a (on shell) $Z$ boson. Yet, for a large part of the IDM parameter space, $\Delta M_{A X}>M_{Z}$, where $A \rightarrow X Z$ proceeds with an on shell $Z$. Therefore, large values of $M_{A}$ not only give smaller cross sections, but also a very large fraction of the yield of leptons are cut. Thus, the prospects for future discovery are slim.

Very recently, ATLAS has also provided limits on the masses of the supersymmetric charginos and neutralinos in the pure (simplified) wino and (nondegenerate) Higgsino limits of the MSSM [136] based on data from the LHC at $\sqrt{s}=13 \mathrm{TeV}$. In these special manifestations of the MSSM (heavy mass sfermions), the production mechanisms and the signatures at the LHC are the same as those of the IDM. The only (notable) difference is the spin of the produced particles in these models. Therefore, we also take into account the ATLAS exclusion zones observing that the corresponding limits on the IDM are more relaxed because of the scalar nature of the IDM particles resulting in much smaller cross sections. In Table II, we therefore also provide the corresponding cross sections for the electroweak production of the IDM alongside those of the wino and Higgsino production processes. Moreover, we also perform a recasting analysis based on MadAnalysis 5 [137-139] on some of the benchmarks points. A more extensive recast of the IDM will be studied independently of this series of papers. We check few of the benchmarks against some of the existing searches. In particular, we use

\footnotetext{
${ }^{8}$ This lower limit depends slightly on the masses of the other scalars. For us, it rests a very good starting point to find viable IDM DM candidates.
} 
TABLE II. Benchmarks points that pass the constraints discussed in Sec. IV. All masses are in GeV. The benchmarks are divided into three classes: 1) the Higgs resonance region, 2) the small co-annihilation region with $\Delta M=M_{A}-M_{X}$ allowed by LEPII and 3) the less tuned annihilation class for masses of a DM around $M_{X}=70 \mathrm{GeV}$. For the relic density, we use micromEGAs $\mathbf{5 . 0 7}$ for which the SM parameters are given in Sec. III B. The relic density is calculated both with an OS $\alpha$ in the Thomson limit and with the effective $\alpha\left(M_{Z}^{2}\right)$. To weigh the dependence of $\lambda_{L}$, we also give the result for $\lambda_{L}=0$. The relative contribution of the most important annihilation/ coannihilation (more than $5 \%$ ) cross sections to the relic density are given: $X X \rightarrow W^{+} W^{-}, Z Z, b \bar{b}, g g$ and $A X \rightarrow f \bar{f}$. These relative contributions do not depend much on the value of the $\alpha$ used for the relic density calculation. For the relative contributions, we therefore show only those calculated with $\alpha(0)$. In the particular case of point P57, the relative contributions are changed drastically when $\lambda_{L}=0$ (they become $90 \%$ into $W W^{\star}$ and $10 \%$ into $Z Z^{\star}$ ) since the Higgs boson mediated $b \bar{b}$ final state is eliminated with $\lambda_{L}=0$. We do not show, in this table, either the values of the $S, T, U$ parameters or of the direct detection. The latter can be trivially rescaled; see Sec. IV for the theoretical constraints of unitarity, which we carefully check. For each point, we do however give the values of the LHC ( $\sqrt{s}=13 \mathrm{TeV}$ with NN23LO1 parton distribution function, pdf, as implemented in MadGraph5_aMC@NLO [149]) cross sections (in fb) for the electroweak production of a pair of the new scalars, should future LHC analyses update the exclusion regions. Alongside these IDM cross sections, we also list the wino and Higgsino cross sections that the LHC Collaborations have studied (simplified wino and nondegenerate Higgsino) [136] and may update in the future. Note that the phenomenology of point A at a high luminosity LHC is considered in [83] and that of point B in [134]. The LHC cross sections are computed at LO. NLO results for the cross sections at the LHC should be scaled by a factor of $30 \%$. While the NLO corrections in the case of supersymmetry have been computed, we expect the same corrections for the IDM, since these are DY-like processes and the corrections are essentially initial-state QCD corrections. We also show the values of the Higgs boson to diphoton signal strength that includes the IDM contribution, should this observable be better constrained in future LHC analyses.

\begin{tabular}{|c|c|c|c|c|c|c|c|c|c|c|c|c|}
\hline & \multicolumn{2}{|c|}{ Resonant Higgs Boson } & \multicolumn{2}{|c|}{ Coannihilation } & \multicolumn{8}{|c|}{$X X \rightarrow W W^{\star}, Z Z^{\star}$} \\
\hline & P57 & P59 & P58 & P60 & A & $\mathrm{B}$ & $\mathrm{C}$ & $\mathrm{D}$ & $\mathrm{E}$ & $\mathrm{F}$ & G & $\mathrm{H}$ \\
\hline$M_{X}$ & 57 & 59 & 58 & 60 & 70 & 70 & 70 & 70 & 72 & 72 & 72 & 70 \\
\hline$\lambda_{L} \times 10^{3}$ & 2.4 & 1.0 & 0.0 & 0.0 & 5.0 & 5.0 & 4.7 & 4.7 & 0.5 & 3.8 & 0.1 & 7.0 \\
\hline$M_{A}$ & 113 & 113 & 66 & 68 & 170 & 130 & 360 & 571 & 165 & 138 & 158 & 250 \\
\hline$M_{H^{ \pm}}$ & 123 & 123 & 110 & 150 & 200 & 240 & 360 & 571 & 165 & 138 & 158 & 250 \\
\hline \multicolumn{13}{|c|}{$\Omega h^{2}$} \\
\hline$\alpha(0)$ & 0.113 & 0.108 & 0.113 & 0.116 & 0.156 & 0.153 & 0.146 & 0.142 & 0.119 & 0.119 & 0.121 & 0.142 \\
\hline$\alpha\left(M_{Z}^{2}\right)$ & 0.118 & 0.113 & 0.101 & 0.103 & 0.130 & 0.128 & 0.121 & 0.119 & 0.099 & 0.099 & 0.101 & 0.119 \\
\hline$\alpha\left(M_{Z}^{2}\right), \lambda_{L}=0$ & 1.97 & 1.97 & 0.101 & 0.103 & 0.146 & 0.143 & 0.135 & 0.132 & 0.100 & 0.107 & 0.102 & 0.140 \\
\hline$\Omega_{W W^{\star}}(\%)$ & 22 & 24 & 5 & 9 & 90 & 90 & 88 & 88 & 89 & 88 & 88 & 88 \\
\hline$\Omega_{Z Z^{\star}}(\%)$ & $\ldots$ & $\ldots$ & $\ldots$ & $\ldots$ & 10 & 10 & 12 & 12 & 11 & 12 & 12 & 12 \\
\hline$\Omega_{b b}(\%)$ & 58 & 57 & $\cdots$ & $\cdots$ & $\cdots$ & $\cdots$ & $\cdots$ & $\cdots$ & $\cdots$ & $\cdots$ & $\cdots$ & $\cdots \cdots$ \\
\hline$\Omega_{g g}(\%)$ & 8 & 7 & $\cdots$ & $\cdots$ & $\cdots$ & $\cdots$ & $\cdots$ & $\cdots$ & $\cdots$ & $\cdots$ & $\cdots$ & $\cdots$ \\
\hline$\Omega_{A X \rightarrow f \bar{f}}(\%)$ & $\cdots$ & $\cdots$ & 95 & 91 & $\ldots$ & $\ldots$ & $\ldots$ & $\ldots$ & $\ldots$ & $\ldots$ & $\ldots$ & $\cdots$ \\
\hline$\mu_{\gamma \gamma}$ & 1.022 & 1.024 & 1.026 & 1.021 & 1.021 & 1.018 & 1.015 & 1.014 & 1.026 & 1.032 & 1.028 & 1.018 \\
\hline \multicolumn{13}{|c|}{ LHC13 cross sections } \\
\hline$A \rightarrow Z X$ open & No & No & No & No & Yes & No & Yes & Yes & Yes & No & Yes & Yes \\
\hline$H^{ \pm} \rightarrow W^{ \pm} X$ open & No & No & No & Yes & Yes & Yes & Yes & Yes & Yes & No & Yes & Yes \\
\hline$p p \rightarrow A X$ & 364 & 352 & 1475 & 1277 & 94 & 198 & 8 & 1 & 100 & 163 & 113 & 29 \\
\hline Higgsino & 4048 & 3894 & 18310 & 15430 & 1019 & 2184 & 86 & 15 & 1088 & 1800 & 1233 & 305 \\
\hline$p p \rightarrow H^{ \pm} X$ & 497 & 481 & 659 & 272 & 104 & 59 & 15 & 3 & 178 & 287 & 201 & 52 \\
\hline Higgsino & 5366 & 5199 & 7196 & 2909 & 1123 & 637 & 161 & 28 & 1931 & 3140 & 2177 & 557 \\
\hline$p p \rightarrow H^{ \pm} A$ & 200 & 200 & 566 & 244 & 37 & 35 & 3 & 0.3 & 58 & 112 & 68 & 12 \\
\hline wino & 8890 & 8890 & 24850 & 10550 & 1756 & 1634 & 138 & 19 & 2666 & 5076 & 3122 & 579 \\
\hline Higgsino & 2222 & 2222 & 6201 & 2642 & 438 & 409 & 35 & 4.6 & 669 & 1274 & 781 & 144 \\
\hline$p p \rightarrow H^{+} H^{-}$ & 97 & 97 & 144 & 48 & 17 & 9 & 2 & 0.4 & 34 & 64 & 39 & 7.3 \\
\hline wino & 3814 & 3814 & 5704 & 1860 & 650 & 328 & 66 & 9 & 1318 & 2523 & 1539 & 280 \\
\hline Higgsino & 1045 & 1045 & 1537 & 521 & 186 & 94 & 19 & 3 & 372 & 699 & 434 & 81 \\
\hline
\end{tabular}

the $2 \ell+\mathbb{E}_{T}$ [140,141] from electroweakinos/slepton pair production, the $2 \ell+\mathbb{E}_{T}, 3 \ell+\mathbb{E}_{T}[142,143]$ from the chargino-neutralino pair production, the $2 \ell+\mathbb{E}_{T}$ $[144,145]$ from the mono- $Z$ search and the $\ell+\mathbb{E}_{T}$ $[146,147]$ from the $W^{\prime}$ search. We must mention here that the topologies considered in the latter two analyses are not the same as ours, and detailed simulations including relevant cuts are necessary for a proper study. However, the aforementioned analyses, especially the ones with the chargino pair or the chargino-neutralino productions, show 
that our benchmark points are safe. We also use MadAnalysis 5's extrapolation code [148] to show that the checked benchmark points are also allowed at the high luminosity run of the LHC (HL-LHC) with an integrated luminosity of $\mathcal{L}=3 \mathrm{ab}^{-1}$.

\section{F. $\boldsymbol{\mu}_{\gamma \gamma}$}

We also check for a possible constraint from the signal strength in the diphoton decay of the SM Higgs boson,

$$
\mu_{\gamma \gamma}=\frac{\operatorname{Br}_{\mathrm{IDM}}(h \rightarrow \gamma \gamma)}{\mathrm{Br}_{\mathrm{SM}}(h \rightarrow \gamma \gamma)},
$$

with $[129,130]$

$$
\mathrm{Br}_{\mathrm{SM}}(h \rightarrow \gamma \gamma)=(2.27 \pm 0.05) \times 10^{-3} .
$$

The ATLAS and CMS limits for $\mu_{\gamma \gamma}$ are given in Refs. [150,151], respectively. We use the standard combination of signal strengths and uncertainties (see [106]) and impose

$$
\mu_{\gamma \gamma}=1.04 \pm 0.1
$$

The IDM contribution has been worked out in [69]. It turns out, see Table II, that the present signal strength constraint on the IDM is not significant. Nonetheless, we list the values of $\mu_{\gamma \gamma}$ for the benchmark points should future LHC analyses improve the limits.

\section{G. Relic density}

The present limit [1] on the relic density abundance is ${ }^{9}$

$$
\Omega h^{2}=0.1197 \pm 0.0022
$$

The experimental uncertainty is less than $2 \%$. This is the main reason it is extremely important that the theoretical prediction be as precise as possible. Once the cosmological model has been set up, in our case, the thermal freeze-out assumption, the major uncertainty is the evaluation of the annihilation cross sections. These cross sections must be evaluated beyond the tree-level approximation. This is the aim of this series of papers for the IDM model. However, to select a few points that pass all other constraints and weigh in the importance of the radiative corrections, we have to be content with a prediction of the relic density based on tree-level cross sections. We use micrOMEGAs 5.0.7 [107, 120-122,152] for computing the relic densities. Some considerations beyond tree level are taken into account

\footnotetext{
${ }^{9}$ We quote the so-called TT (Planck high multipole TT likelihood) + lowP (low multipole polarization data). Adding external, + "lensing,"... data do not change the limit appreciably, especially in view of the larger theoretical uncertainty that we include.
}

in micrOMEGAs; in particular, the Higgs couplings to the SM particles. The code also uses a running for the electromagnetic coupling with an effective coupling estimated at $\alpha\left(M_{Z}\right) \sim 1 / 128.907$ instead of the OS coupling in the Thomson limit, i.e., $\alpha=/ 137.036$. For many annihilation cross sections, this change of $\alpha$ amounts to an overall rescaling that brings a correction of about $13 \%$ in $2 \rightarrow 2$ annihilation processes. However, in many scenarios and models beyond the SM, this rescaling does not account for the full one-loop correction $[3,5,6,10,106]$. It is therefore wise when we select benchmarks points, using micrOMEGAs for the relic density constraint, to allow for a theoretical uncertainty of $20 \%$. We will therefore keep points that satisfy

$$
0.096<\Omega h^{2}<0.144
$$

Moreover, we quote the value of the relic density using micrOMEGAs with both an effective $\alpha\left(M_{Z}\right)$ and $\alpha$. Once we compute the full one-loop corrections, we comment on the use of the effective electromagnetic coupling for each of the benchmark points that we study at one loop. When running micrOMEGAs, we also let the code check explicitly for the direct detection and possible indirect detection constraints.

\section{THE DIFFERENT CHANNELS CONTRIBUTING TO THE RELIC DENSITY FOR $55<M_{X}<75 \mathrm{GeV}$}

\section{A. Characteristics of the benchmarks points}

Keeping all the constraints that we have introduced so far, we do of course recover the general conclusions of a scan of the IDM with the relic density constraint calculated with micrOMEGAs. Namely, apart from a region with the heavy scalar, $M_{X}>500 \mathrm{GeV}$, scenarios that we investigated in [10], the low mass region range lies in the range $55 \mathrm{GeV}<M_{X}<75 \mathrm{GeV}$.

Within this relatively small range, three different mechanisms are at play,

(i) The coannihilation region

For $M_{X} \sim M_{A}$ (within $M_{X}-M_{A}<8 \mathrm{GeV}$ set by the LEPII constraint), extremely efficient coannihilation $X A \rightarrow f \bar{f}$ takes place. It is so efficient that the Boltzmann factor is needed to reduce its contribution. Therefore, $\Delta M_{A X}$ should be as large as possible. This is the reason this scenario occurs at the very edge of the limit allowed by LEPII, i.e., $M_{A} \sim M_{X}+8 \mathrm{GeV}$. For $M_{X} \sim 55 \mathrm{GeV}, X X$ annihilations are still away from the $h$ peak, and the onset of $X X \rightarrow W f \bar{f}^{\prime}, X A \rightarrow f \bar{f}$ is the principal channel. In any case, for the smallest $M_{X}$ masses, one still needs to reduce annihilation to the SM Higgs boson by setting $\lambda_{L} \sim 0$. Although we find this scenario extremely fine-tuned, we find it a good example for the effect of the loop corrections in a certain limit. Indeed, in this limit, the tree-level process is 
governed totally by the gauge coupling. The oneloop corrections to $A X \rightarrow Z \rightarrow f \bar{f}$ are technically the easiest to consider. A detailed investigation is conducted in Ref. [111].

(ii) The SM Higgs resonance

For $M_{X} \sim M_{h} / 2$, efficient annihilation is possible through the very narrow Higgs resonance. Again, this is also fine-tuned. Nonetheless, the annihilation does require nonzero $h X X, \lambda_{L}$, coupling. The oneloop corrections here will have to deal with the implementation of the width at one loop, a nontrivial problem. We leave all technicalities and discussion on the results in Ref. [110].

(iii) The annihilation $X X \rightarrow W W^{\star}, Z Z^{\star}$

For $M_{X}<80 \mathrm{GeV}$, these annihilations occur with large enough rates even though we are below the $W W$ threshold. They become too efficient when this $W W$ threshold is crossed. Past about $M_{X} \sim 73 \mathrm{GeV}$, the annihilations become too large, depleting the dark matter density. Technically, the one-loop corrections here are quite challenging, requiring the computation of $2 \rightarrow 3$ processes at one loop. We leave these calculations and their impact on the relic density to a separate paper [112]. It is not possible to attempt a $2 \rightarrow 2, X X \rightarrow W f \bar{f}^{\prime}$, with an off shell $W$, and integrate over Breit-Wigner distribution without getting into trouble with gauge invariance. Nonetheless, since $W W$ and $Z Z$ annihilation cross sections are a backbone to the $X X \rightarrow V f \bar{f}$ [112], we study these $2 \rightarrow 2, X X \rightarrow W^{+} W^{-}, Z Z$ on shell processes, see Sec. VII, before we embark on the full $2 \rightarrow 3$ processes. This investigation helps unravel some characteristics of the corrections and more importantly, reveals that one-loop perturbativity may break down for some choices of the parameters that tree-level unitarity allows. This study above the $W W$ and $Z Z$ threshold will help select further among the benchmark points.

These benchmark points based on the constraints we reviewed so far are listed in Table II. Most of the regimes are, at tree level, driven by the gauge coupling but the contribution of $\lambda_{L}$ is not negligible. The value of $\lambda_{L}$ plays a major role in the derivation of the cross section for the Higgs resonance scenario since this mechanism calls for the $h X X$ coupling. The coannihilation region requires $\lambda_{L} \simeq 0$ and is therefore driven by the gauge coupling. While the (nonresonant) annihilation region, $X X \rightarrow W f \bar{f}^{\prime}$ and $X X \rightarrow Z f \bar{f}$, is dominated by the gauge coupling, the $\lambda_{L}$ contribution is not negligible at all. First, it occurs because there is a contamination from the SM Higgs exchange but also because the production of longitudinal vector bosons is $\lambda_{L}$ dependent. This $\lambda_{L}$ dependence can be seen in the $X X G G$ and $X X G^{+} G^{-}$quartic couplings, which once the masses of the scalars are fixed ( $\lambda_{4,5}$ fixed), the coupling is sensitive to $\lambda_{L}$. Table II gives the value of the relic density
TABLE III. Values of the underlying $\lambda_{3,4,5}$ parameters and the corresponding $\beta$ functions for $\lambda_{L}, \tilde{\beta}_{\lambda_{L}}$, for the benchmarks points defined in Table II.

\begin{tabular}{lcccc}
\hline \hline Model & $\lambda_{4}$ & $\lambda_{5}$ & $\lambda_{3}$ & $\beta_{\lambda_{L}}$ \\
\hline P57 & -0.227579 & -0.152146 & 0.382125 & $1.12312+2.15628 \lambda_{2}$ \\
P59 & -0.223871 & -0.148438 & 0.373309 & $1.09738+2.09499 \lambda_{2}$ \\
P58 & -0.263378 & -0.015854 & 0.279232 & $0.77300+1.18034 \lambda_{2}$ \\
P60 & -0.587742 & -0.016365 & 0.604108 & $1.50953+2.48189 \lambda_{2}$ \\
A & -0.738354 & -0.383561 & 1.12691 & $4.34278+6.0819 \lambda_{2}$ \\
B & -1.49269 & -0.19178 & 1.68947 & $7.2441+7.565 \lambda_{2}$ \\
C & -1.99292 & -1.99292 & 3.99053 & $51.1306+23.9714 \lambda_{2}$ \\
D & -5.13238 & -5.13238 & 10.2695 & $324.269+61.6449 \lambda_{2}$ \\
E & -0.352252 & -0.352252 & 0.705005 & $2.48707+4.23303 \lambda_{2}$ \\
F & -0.221506 & -0.221506 & 0.446813 & $1.40176+2.70368 \lambda_{2}$ \\
G & -0.316118 & -0.316118 & 0.632336 & $2.14181+3.79461 \lambda_{2}$ \\
H & -0.920545 & -0.920545 & 1.84809 & $12.0397+11.1305 \lambda_{2}$ \\
\hline \hline
\end{tabular}

when $\lambda_{L}$ is switched off. Because of the importance of this coupling and the fact that we will, in some cases, rely on an $\overline{\mathrm{MS}}$ scheme for its renormalization, we give in Table III the value of the $\beta$ constant of $\lambda_{L}, \tilde{\beta}_{\lambda_{L}}$, for each benchmark point. Observe the very large values we obtain for this constant for points $\mathrm{C}$ and $\mathrm{D}$ that are associated with large values of the heavy scalars.

\section{B. Cross sections and velocity dependence}

The following subsection is too well known for DM practitioners, but we hope it will be of relevance to our colleagues, experts in NLO calculations who want to apply their know how to DM annihilation. Moreover, since the calculation of the relic density needs that one generates data at NLO for many values of the relative velocity and that the result of each relative velocity is time consuming, it helps to know where NLO data should most judiciously be generated for DM relic abundance prediction.

In order to convert the cross sections into their contribution to the relic abundance, a thermodynamical/cosmological model needs to be implemented. In the case of freeze-out that we will be working with, a thermal average and a convolution of the cross sections over relative velocities of the annihilating particles are performed. The velocity distribution is important since if the cross sections are large in regions where the weight of the velocity distribution is negligible, the contribution of the process to the relic density is very much reduced. In case of annihilations, $v$ determines the centre of mass total energy, or the kinematical invariant $s$, as

$$
v=2 \sqrt{1-\frac{4 M_{X}^{2}}{s}} \quad \text { or } \quad \sqrt{s}=2 M_{X} \frac{1}{\sqrt{1-\frac{v^{2}}{4}}}
$$


Although we will interface all of our cross section to micrOMEGAs for the velocity and temperature averaging for a full numerical integration, it is instructive to gain an analytical understanding. The velocity averaged cross section, at a temperature $T$ for annihilating DM particles of mass $M_{X}$ can be approximated as

$$
\begin{aligned}
\langle\sigma v\rangle= & \int_{0}^{\infty} d v f(v, x)(\sigma v) ; \quad f(v, x)=\frac{x^{3 / 2}}{2 \sqrt{\pi}} v^{2} e^{-x v^{2} / 4} \\
& x=M_{X} / T,(x>1) .
\end{aligned}
$$

Figure 1 shows the weight function of the velocity distribution at the freeze-out temperature, $T_{f}$. As a general rule, freeze-out occurs at $x_{f}=M_{X} / T_{f} \sim 20-25$. This helps understand which range in the relative velocity, $v$, we need to generate most of the annihilation cross sections. This is particularly useful for the calculations at one-loop cross sections where there is no need to compute the (involved) one-loop cross sections for too many values of $v$ where these cross sections are small. Observe that a typical $v$ where we must get the cross section right is $v \sim 0.4$ (see Fig. 1) and that for $v>0.8$, the cross sections are weighted down. As we will see, there may be exceptions to this general rule [110], in particular, if the cross section is dominated by a peak (in our case, the Higgs resonance region) that may occur toward the higher end of the distribution function and also, to a lesser extent, if the cross section grows because of the opening up of a threshold. In our analyses, for the most general case, we compute the cross sections for values of $v$ as high as $v=0.9$ (or even $v \sim 1$ ). Going to such high values of $v$ makes sense only if the cross section peaks dramatically as

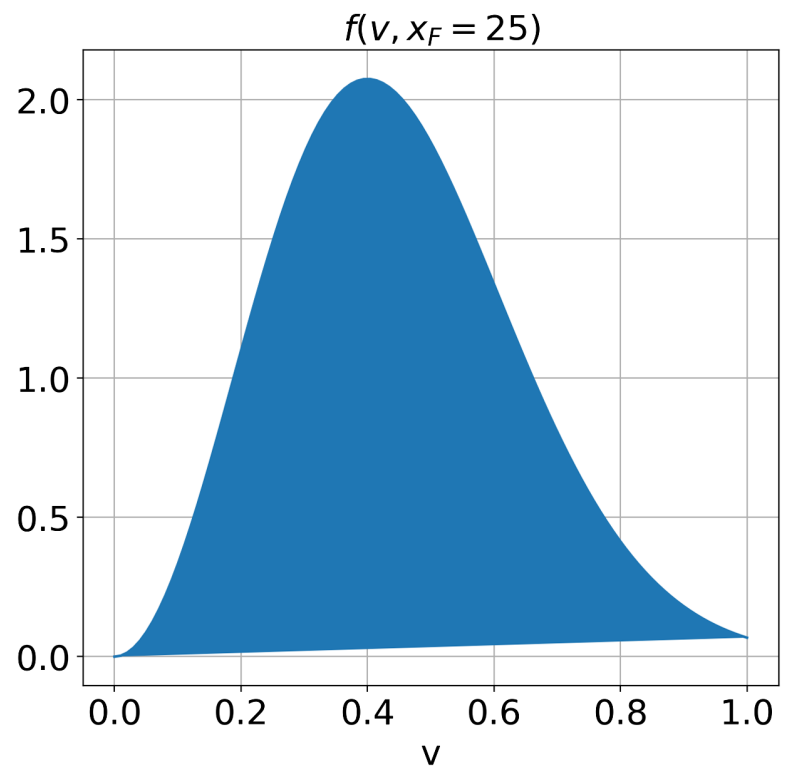

FIG. 1. The normalized velocity distribution $f\left(v, x_{F}=25\right)$ as a function of $v$. is the case of the resonance through the very narrow SM Higgs boson. Indeed, for the point $M_{X}=57 \mathrm{GeV}$, $v=0.82$ corresponds to $\sqrt{s} \simeq M_{h}=125 \mathrm{GeV}$. Observe that for $M_{X}=70,72 \mathrm{GeV}, v=0.8$ does not permit the production of both $W \mathrm{~s}$ on shell but only a $W W^{\star}$, and therefore, we must consider $W f \bar{f}$.

In the case of coannihilation, the Boltzmann factor is a very penalising factor. In our case, this concerns $A X$ coannihilation when the mass difference between these two particles is small. The effective relative weight (to the annihilation $X X$ rate) is

$\tilde{g}_{\mathrm{eff}}=(1+\delta)^{3 / 2} e^{-x \delta}, \quad \delta=m_{-} / M_{X}, \quad m_{ \pm}=M_{A} \pm M_{X}$.

For large $\delta$, coannihilation is therefore exponentially suppressed, and it effectively does not take place. If $\delta$ is too small and the associated coannihilation cross sections are large, we then have too small $\Omega_{\mathrm{DM}}$. In the case of coannihilation, the relative velocity is calculated from the invariant $s$ of the coannihilating particles $X$ and $A$ as

$$
v=2 \sqrt{\left(1-m_{+}^{2} / s\right)\left(1-m_{-}^{2} / s\right)} \frac{1}{1-\frac{m_{+}^{2} m_{-}^{2}}{s^{2}}} .
$$

For $M_{A}=M_{X}\left(m_{-}=0\right)$, we recover the usual annihilation relative velocity of Eq. (5.1). As remarked earlier, in Sloops when calculating the annihilation cross for DM, the phase space factor is modified such that the code returns $\sigma v$ rather than $\sigma$, which could be ill defined for $v=0$. We give the DM annihilation $\sigma v$ cross sections in units of $\mathrm{cm}^{3} \mathrm{~s}^{-1}$. Our conversion factor to translate $\sigma v$ expressed in $\mathrm{GeV}^{-2} \mathrm{~m} / \mathrm{s}$ is

$c_{0}=2.99792 \times 3.8937966 \times 10^{8} \simeq 1.16732 \times 10^{9}$.

\section{THE DIFFERENT STEPS IN THE CALCULATION OF THE RELIC DENSITY WITH RATES AT ONE LOOP}

The annihilation processes we consider are, at tree level, of the type $2 \rightarrow n$ with $n=2$ for annihilation/coannihilation to light fermions or to on shell $W W / Z Z$ pairs and $n=3$ for the type $W W^{\star} / Z Z^{\star}$ for off shell $W / Z$ for which the associated full fermion pair $l \bar{l}^{(\prime)}$ is considered. Even with the help of automatic codes for the calculation of the different components involved in the evaluation of the relic density, at one loop, the task is almost daunting because for each space point in parameter space we need to generate a large number of data corresponding to a large number of relative velocities, $v$, values because many diagrams are generated as we will see in the next section even for a 
relatively simple $2 \rightarrow 2$ subprocess. The different steps we take, for each process, are as follows:

(i) Calculate the tree-level annihilation cross sections for a large set of relative velocities. A first check is made with the code CalcHEP [153], which is used internally in micrOMEGAs. At tree level, the cross sections do not depend on the parameter $\lambda_{2}$. The analysis of the tree-level cross section will indicate how to sample the $v$ values.

(ii) Calculate the one-loop virtual corrections within a specific renormalization scheme, for the same set of relative velocities as at tree level. Generate this step with at least three values of the parameter $\lambda_{2}$. At this step, for processes involving charged (final) particles, the cross sections involve infrared QED divergences which are regulated with a finite fictitious photon mass, $m_{\gamma}$. We check the result of the one-loop virtual corrections by confirming that it is ultraviolet finite and that it is gauge parameter independent by varying the nonlinear gauge parameters (see III C) for a random point in phase space (the latter is a very powerful test).

(iii) The QED infrared divergences from the virtual oneloop corrections cancel out against the ones emanating from the real radiation after integration over the photon spectrum in the (tree level) reaction $2 \rightarrow n+\gamma$, which we generate. Here we use the slicing technique (see, for example, [154]) by slicing the energy of the outgoing photon $E_{\gamma}$ at $E_{\gamma}=k_{c} . k_{c}$ is chosen sufficiently soft, such that the factorization of soft photons (with the same fictitious photon mass as the one chosen for the virtual corrections) is applicable. Integration over $E_{\gamma}$, for $E_{\gamma}>k_{c}$, is carried out numerically for different values of $k_{c}$. This step is time consuming since one must make sure the final result (virtual one-loop + real photon radiation) does not depend on $k_{c}$ beside being independent of $m_{\gamma}$.

(iv) Once the set of corrected one-loop (virtual and real radiation) $v$ dependent cross sections is generated, all annihilation subprocess that contribute to the relic density are passed as a table to micrOMEGAs substituting the default micrOMEGAs tree-level $v$ annihilation cross sections tables. micrOMEGAs has a built-in interface for such a purpose. Among other possibilities, the users can replace (or/and remove) any default cross section by their own. At this stage, some sanity checks are performed, as we will explain in more detail for the difficult Higgs resonance in Ref. [110].

We stress that we leave the (numerical) resolution of the Boltzmann equations to micrOMEGAs. We therefore implicitly assume the same (standard freeze-out) assumptions (full equilibrium, no entropy production...) that go into the calculation of the relic density. Even if the full $\mathcal{O}(\alpha)$ correction we plug into micrOMEGAs contains, for charged final states, a hard photon part, the latter dresses the final charged particle and thermodynamically (from the point of view of the Boltzmann equations), it is classified as a $2 \rightarrow 2$ process. Therefore, the use of micrOMEGAs is totally justified. Moreover, by comparing the full NLO corrections of the charged channels and the corresponding neutral channel, where there is no QED correction (for example, comparing $A X \rightarrow \mu^{+} \mu^{-}$vs $A X \rightarrow \nu \bar{\nu}$ ), the effect of the hard photon is negligible, as we will see in [111].

Observe that the $2 \rightarrow 3$ processes $X X \rightarrow W f \bar{f}^{\prime}$ represent a gauge invariant process to correctly calculate $X X \rightarrow W W^{\star}$ below threshold, a cross section which is not negligible at all. For early computations of such below threshold processes and their impact on the relic (freeze-out) density, see Refs. [155-158]. This type of processes is now taken care of by micrOMEGAs as generalized $2 \rightarrow 2$ processes for the implementation of the Boltzmann equations. The full calculation, both at tree level, and at one loop, shows [110] that the flavor dependence of the $X X \rightarrow W f \bar{f}^{\prime}$ is very well described by the branching fraction $\operatorname{Br}\left(W \rightarrow f \bar{f}^{\prime}\right)$. Therefore, the one-loop implementation is like that of the tree-level implementation as concerns the Boltzmann equations in micrOMEGAs. The same applies to the $X X \rightarrow Z f \bar{f}$.

It may be argued that the very small coupling of the SM Higgs boson to DM required to obtain the present experimental value of the relic density, thanks to the boost from the annihilation at the very narrow Higgs resonance, implies a suppression of the rate of DM scattering off the particles in the bath signaling (early) kinetic decoupling, which is a departure from the usual assumption of the freeze-out mechanism $[159,160]$. If this were the case, a phenomenological study should include this effect, which at the moment is not included in micrOMEGAs. However, in the present series, we concentrate on how to precisely set up and calculate the one-loop corrections for such a tricky situation. We have also given the corrected annihilation cross sections (with their $v$ dependence) so they could be interfaced with some other code than micrOMEGAs.

\section{VII. $X X \rightarrow Z Z, W^{+} W^{-}$AT ONE-LOOP AS A WARM-UP}

The $X X \rightarrow Z Z, W^{+} W^{-}$annihilations, with both vectors on shell, are too efficient to account for a relic density in accordance with observation within the standard cosmological model of freeze-out. This is the reason that DM masses above $80 \mathrm{GeV}$ do not feature in Table II. However, many of the benchmark points in Table II survive because if one of the vector bosons is off shell, the cross sections are no longer so large. The $2 \rightarrow 3$ cross sections $X X \rightarrow W f \bar{f}^{\prime}$ and $X X \rightarrow Z f \bar{f}$ do carry the salient features of the on shell production. It is much more transparent to investigate these features on the $2 \rightarrow 2$ process rather than on the more 
technically challenging $2 \rightarrow 3$ processes that we will study in Ref. [112]. Besides, $X X \rightarrow W^{+} W^{-}, Z Z$ for $M_{X}>$ $80 \mathrm{GeV}$ may still be a viable model since it does not lead to overabundance but would not account for all of DM. It would then be supplemented by a new ingredient to the IDM. In any case, our aim here is to unravel some important characteristics of these $2 \rightarrow 2$ cross sections.

Because of $S U(2)$ symmetry, $X X \rightarrow W^{+} W^{-}$and $X X \rightarrow Z Z$ share common features. At one loop, we will concentrate first on $X X \rightarrow Z Z$ to bring up the most important features that will help later understand both our full one-loop calculation of both $X X \rightarrow W f \bar{f}^{\prime}$ and $X X \rightarrow Z f \bar{f}$. We do this because $X X \rightarrow Z Z$ is easier to compute since there is no need to consider real corrections of the sort $X X \rightarrow W^{+} W^{-} \gamma$ that are necessary to regulate the infrared divergences of the virtual corrections. We take $M_{X}=100 \mathrm{GeV}$ and require all constraints of Sec. IV to hold, apart from the relic density within the Planck limit. Independently of the direct detection limit, we keep $\lambda_{L}$ very small, so that we are in a similar situation as in the $2 \rightarrow 3$ processes that we will study and whose characteristics are given by the benchmark points. For the same reason, the different choices of $M_{A}, \lambda_{L}$ correspond to $\lambda_{3}, \lambda_{4}, \lambda_{5}$ values similar to those found in the benchmark points for $X X \rightarrow W W^{\star}, Z Z^{\star}$. At one loop, one needs to specify the value of $\lambda_{2}$. For each point, we take the values $\lambda_{2}=0.01$, 1,2 . These are the same values we considered in our study of the heavy mass IDM [10]. The aim of this introductory analysis serves to understand the following key points.

(i) Since we are in scenarios where $M_{h}<2 M_{X}$ and can not take an OS definition of $\lambda_{L}$ based on the input $\Gamma_{h \rightarrow X X}$, we have to rely on the $\overline{\mathrm{MS}}$ renormalization scheme. We therefore want to investigate how large the scale dependence of the scheme is and whether we can advocate a choice for the optimum scale.

(ii) Is perturbativity maintained at one loop? Could the one-loop radiative corrections turn out to be too large for certain combinations of the underlying parameters?

(iii) How much do the corrections depend on $\lambda_{2}$. If the impact of $\lambda_{2}$ is not small, phenomenological considerations based on tree-level analyses should be reconsidered.

(iv) As an aside, we can ask whether there could be circumstances when a running of $\alpha$ (defined at $M_{Z}$ as assumed in micrOMEGAs) could account for the bulk of the corrections regardless of the value of $\lambda_{2}$.

(v) The scale dependence and the $\lambda_{2}$ dependence were touched upon in our study of the heavy mass IDM [10], and we promised to investigate these issues further. We will see here how the scale dependence can be derived quantitatively and how the $\lambda_{2}$ dependence is not all contained in $\tilde{\beta}_{\lambda_{L}}$.

Answers to these questions will guide us when we study the $2 \rightarrow 3$ processes. Note that we investigate the first three items above in our study of $\Gamma_{h \rightarrow X X}$ in the $\overline{\mathrm{MS}}$ scheme in Sec. III G.

To unravel some key features of the one-loop corrections, we consider five test points. While the DM mass, $M_{X}$, is fixed at $M_{X}=100 \mathrm{GeV}$ for all test points, we consider different values of $\lambda_{3,4,5}$ with the constraint that $\lambda_{L} \ll 1$. This allows us to generates different values for $\beta_{\lambda_{L}}$ as well as different values of $M_{A, H^{ \pm}}$with nonetheless $M_{A} \simeq M_{H^{ \pm}}$ (within the $T$ parameter constraint). Characteristics of the test points are listed in Table IV.

\section{A. $X X \rightarrow Z Z, W^{+} W^{-}$at tree level. The $\lambda_{L}$ dependence}

The investigation of the tree-level cross section gives us the $\lambda_{L}$ dependence, which will then determine the scale dependence at one loop in the $\overline{\mathrm{MS}}$. The $\lambda_{L}$ dependence will, at one loop, track the scale dependence, which is only carried by the counterterm $\delta \lambda_{L}$.

At tree level, the $X X \rightarrow Z Z$ Born amplitude is built up from the $s$-channel SM Higgs exchange, the quartic $X X Z Z$ interaction, and the exchange of $A$ in the $t$ channel; see Fig. 2. For $X X \rightarrow W^{+} W^{-}$, it is $H^{ \pm}$, which is involved in the $t$-channel exchange. The tree-level cross sections for $X X \rightarrow W^{+} W^{-}$and $X X \rightarrow Z Z$ for test point $\mathrm{P} 1$ are shown in Fig. 3. Test point $\mathrm{P} 1$ is characterized by $\lambda_{L}=10^{-4}$ and $M_{X}, M_{A}, M_{H^{ \pm}}=100,120,130 \mathrm{GeV}$. The ratio between the $W W$ cross section and the $Z Z$ cross section is almost constant and only changes by about $6 \%$ in the range $v=0-0.9$ (and less than 3\% in the most relevant range $v=0-0.4$ ), as shown in Fig. 3. For smaller $v, X X \rightarrow Z Z$ is slightly impacted by the threshold factor (for $M_{X}=$ $100 \mathrm{GeV}, Z Z$ is closer to the threshold than $W^{+} W^{-}$), which explains why the ratio is higher for small $v$ than for larger $v$ s. The tree-level behavior helps understand why these two cross sections will share common features also at one loop.

As we pointed out before, the $\lambda_{L}$ dependence is not due to Higgs exchange only, but it intervenes also in the $X X Z Z$ quartic coupling. At the cross section level, these contributions lead to the quadratic $\lambda_{L}^{2}$ dependence. The interference of these contributions with the $t$-channel amplitudes leads to a (linear) $\lambda_{L}$ dependence in the cross section. For each point in parameter space and for any given energy, or relative velocity, the $\lambda_{L}$ dependence of the tree-level cross section, $\sigma_{\text {tree }}$, can then be written as

$$
\sigma_{\text {tree }}=\sigma_{0}+\lambda_{L} \sigma_{1}+\lambda_{L}^{2} \sigma_{2}
$$

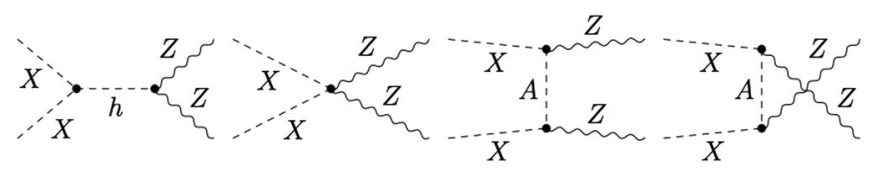

FIG. 2. Born contributions diagrams to $X X \rightarrow Z Z$. 

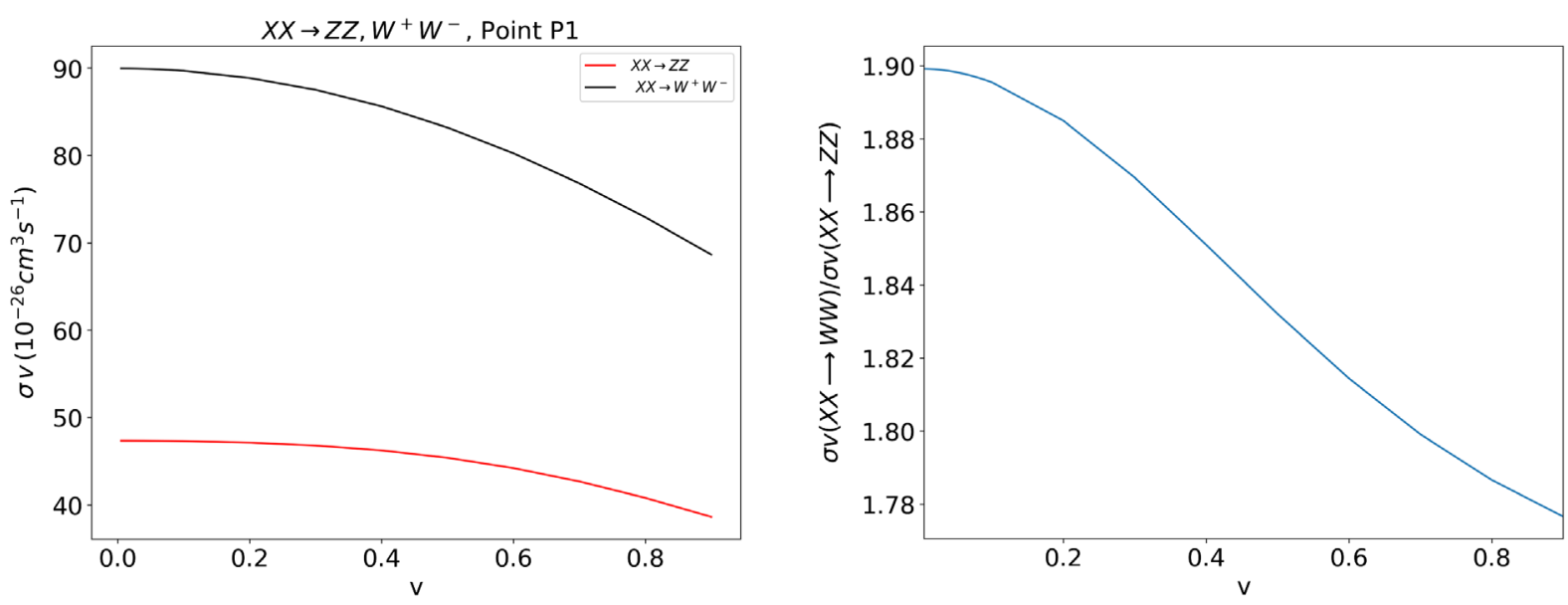

FIG. 3. The ratio $\sigma v\left(X X \rightarrow W^{+} W^{-}\right) / \sigma v(X X \rightarrow Z Z)$ as a function of the relative velocity, $v$, shown here for model P1.

For $X X \rightarrow Z Z$, both the Higgs exchange and the $X X Z Z$ quartic coupling contribute to $\sigma_{2}$, whose value depends on $M_{X}$ and $M_{h}$ given a specific relative velocity. The $t$-channel $A$ exchange diagrams and part of the $X X Z Z$ contribute to $\sigma_{0}$ and depend therefore on $M_{A}$ (and the SM gauge coupling). $\sigma_{1}$ is the result of the interference of the amplitudes contributing to $\sigma_{2}$ and $\sigma_{0}$. We choose to derive $\sigma_{0,1,2}$ numerically from Sloops. For this purpose, we generate three values of $\sigma_{\text {tree }}$ corresponding to three values of $\lambda_{L}$ (we take $\lambda_{L}=0,1,2$ ) with all other parameters fixed. We are then able to reconstruct the $\lambda_{L}$ dependence in Eq. (7.1) from a fit. Checks of the fit were found to be excellent when comparing the result of the fit-cross section with a direct (tree-level) calculation for a fourth value of $\lambda_{L}$ not used for the fit. Obviously, for this $2 \rightarrow 2$ process, the $\lambda_{L}$ dependence could have been derived analytically but the main reason we propose this procedure is because we also use this procedure for the $2 \rightarrow 3$ processes [112], where an analytical expression is rather involved.

\section{B. $X X \rightarrow Z Z$ at one loop. Some examples}

We start by considering $X X \rightarrow Z Z$ for a fixed value of $v$, $v=0.4$, before showing the full one-loop corrections for both $X X \rightarrow Z Z$ in the range $0<v<0.9$. At the heart of our discussion is the $\lambda_{L}$ dependence of the cross sections, which we construct according to Eq. (7.1).

$X X \rightarrow Z Z$ at one loop shows a very a rich structure that accesses the parameters of the full model, in particular the $\lambda_{2}$ parameter of the dark sector. The latter shows up as rescattering effects $X X \rightarrow A A$ and $X X \rightarrow X X$; see Fig. 4.

Before showing the results of the full one-loop corrections in the $\overline{\mathrm{MS}}$ scheme, let us derive the scale variation from $\lambda_{L}$ dependence of the tree-level cross section, $\sigma_{\text {tree }}$ (see (7.1). The scale variation only enters through the scale variation of $\lambda_{L}, \partial \lambda_{L} / \partial \log \mu=-\beta_{\lambda_{L}} / 16 \pi^{2}$. Since

$$
\delta \sigma_{\text {tree }}=\frac{\partial \sigma}{\partial \lambda_{L}} \delta \lambda_{L}=\left(\sigma_{1}+2 \lambda_{L} \sigma_{2}\right) \delta \lambda_{L},
$$

one can relate the one-loop correction, $\delta \sigma$, between two scales,

$\delta \sigma\left(\bar{\mu}_{2}\right)=\delta \sigma\left(\bar{\mu}_{1}\right)-\frac{1}{16 \pi^{2}}\left(\sigma_{1}+2 \lambda_{L} \sigma_{2}\right) \tilde{\beta}_{\lambda_{L}} \ln \left(\bar{\mu}_{2} / \bar{\mu}_{1}\right)$.

Equation (7.3) agrees perfectly with the numerical results of the full one-loop corrections we obtain with our code. The results are presented in Table IV. We could have run the code for only one value of $\mu_{\mathrm{dim}}$ and derived the results for any other scale through Eq. (7.3). However, extracting the results for the different scales from the code is testimony that the code works very well, and the implementation is consistent.

For a later reference, with the condition that $\lambda_{L} \ll 1$, note that in terms of relative correction (normalized to the treelevel cross section), Eq. (7.3) turns into

$$
\begin{aligned}
& \frac{\delta \sigma\left(\bar{\mu}_{2}\right)}{\sigma_{\text {tree }}}=\frac{\delta \sigma\left(\bar{\mu}_{1}\right)}{\sigma_{\text {tree }}}-\frac{1}{16 \pi^{2}}\left(\frac{\sigma_{1}+2 \lambda_{L} \sigma_{2}}{\sigma}\right) \tilde{\beta}_{\lambda_{L}} \ln \left(\bar{\mu}_{2} / \bar{\mu}_{1}\right) \\
& \frac{\delta \sigma\left(\bar{\mu}_{2}\right)}{\sigma_{\text {tree }}} \simeq \frac{\delta \sigma\left(\bar{\mu}_{1}\right)}{\sigma_{\text {tree }}}-\frac{1}{16 \pi^{2}} \frac{\sigma_{1}}{\sigma_{0}} \tilde{\beta}_{\lambda_{L}} \ln \left(\bar{\mu}_{2} / \bar{\mu}_{1}\right) .
\end{aligned}
$$

Equation (7.4) indicates that the scale variation and the associated correction is large when $\tilde{\beta}_{\lambda_{L}}$ is large but also when $\sigma_{1} / \sigma_{0}$ is large. For the test points $\mathrm{P} 0-\mathrm{P} 5, \sigma_{1} / \sigma_{0}$ is between 5 and 6 .

Our results for the full one-loop corrections of the cross section $X X \rightarrow Z Z$ for points $\mathrm{P} 0-\mathrm{P} 5$, evaluated at $v=0.4$, are given in Table IV. Beside listing the characteristics of the points $\mathrm{P} 0-\mathrm{P} 5$, Table IV provides the $\lambda_{L}$ dependence of the tree-level cross sections as well as the $\tilde{\beta}_{\lambda_{L}}$ of the model such that the reader can compare the results of the analytical scale variation [Eq. (7.4)] with the numerical output of Sloops for the full one-loop corrections for the different renormalization scales and values of $\lambda_{2}$. In passing, note that the relative dependence of the cross section in $\lambda_{L}$ is not large. This dependence will be stronger 

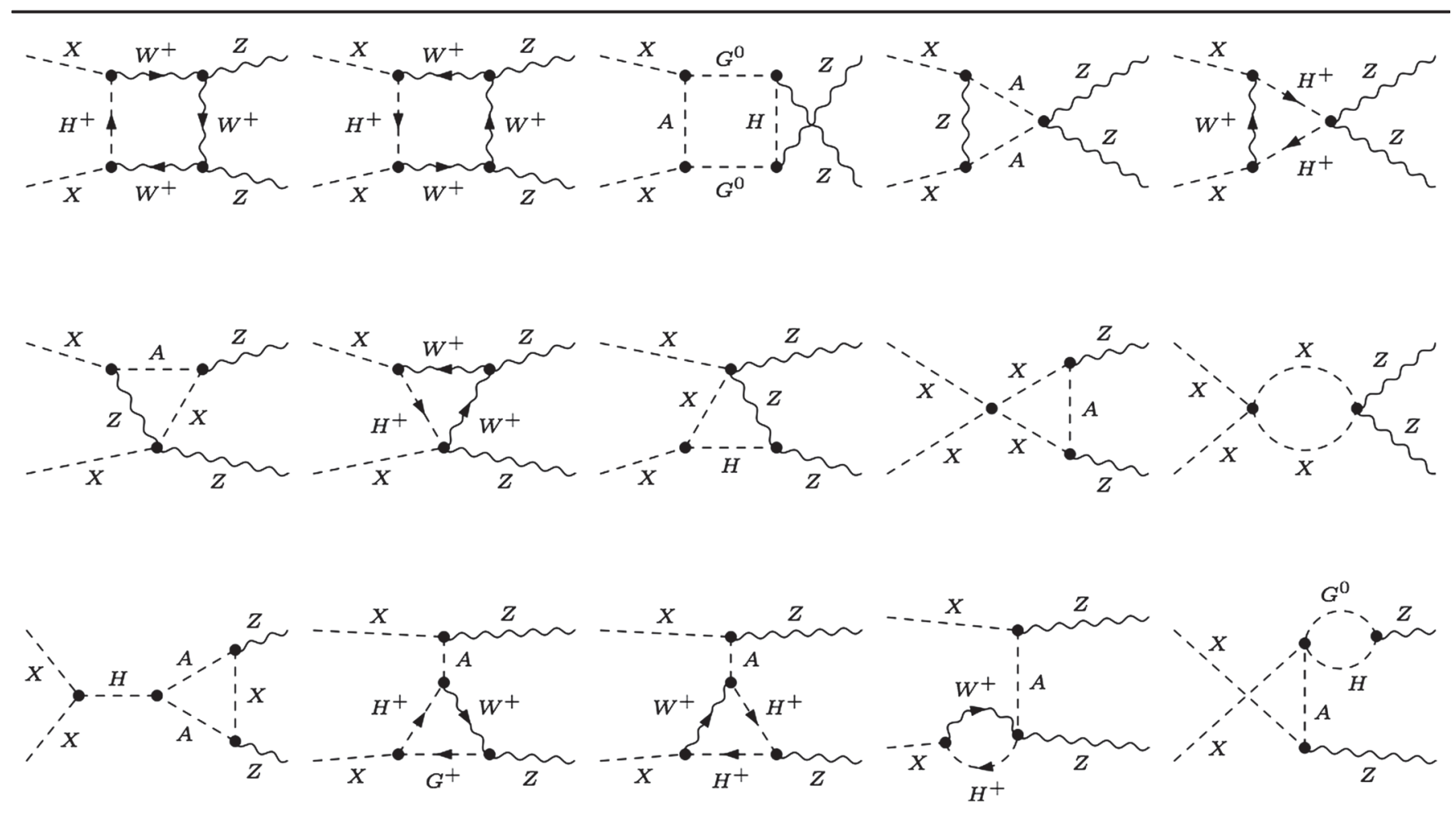

FIG. 4. A small selection of one-loop contribution diagrams to $X X \rightarrow Z Z$. We only picked up some box and triangle contributions. Note the rescattering $X X \rightarrow X X$ and $X X \rightarrow A A$ diagrams are solely within the dark sector (last two diagrams in the second row and the last diagram in the third row).

when we study $2 \rightarrow 3$ processes [112]. Table IV is extremely instructive. It reveals many important points.

(i) The rescattering effects within the dark sector, through the $\lambda_{2}$ dependence, are confirmed. The one-loop calculation shows that $\lambda_{2}$ enters the prediction of the annihilation cross section not only through the running of the coupling $\lambda_{L}$, through $\tilde{\beta}_{\lambda_{L}}$, but there is also an added genuine one-loop contribution. Indeed, we have chosen test point P0 because its $\tilde{\beta}_{\lambda_{L}}$ does not depend on $\lambda_{2}$. Therefore, the results for P0 do show a dependence of the correction on $\lambda_{2}$ that is not an effect of the scale dependence of $\lambda_{L}$. For P0, this dependence can be derived easily from Table IV, by taking the difference of any two columns (that is the difference between two values of $\lambda_{2}$ evaluated at the same scale). We find that $\delta \sigma\left(\lambda_{2}\right)=-2.56 \lambda_{2}$ or in terms of relative correction $-6.5 \lambda_{2}(\%)$, which is not negligible for values of $\lambda_{2}>1$. For the other test points, there is also a $\lambda_{2}$ dependence that enters through $\tilde{\beta}_{\lambda_{L}}$. Therefore, only a full one-loop correction captures the complete $\lambda_{2}$ dependence, beyond the dependence contained in $\tilde{\beta}_{\lambda_{L}}$. The $\lambda_{2}$ dependence is important.

(ii) The running of $\alpha$ at the scale of the process, of order $M_{Z}$, does not account for the full one-loop correction. It can be considered as an acceptable description only for an almost vanishing $\lambda_{2}$ and very small $\lambda_{3,4,5}$. In fact, for the test point P0 with $\lambda_{2}=2$, the corrections practically vanish showing that extra corrections totally offset the correction from the running of $\alpha$. Therefore, the use of a running $\alpha$ is of very limited applicability.

(iii) As the split between the DM mass and the mass of the other scalars increases, the one-loop correction and the scale dependence increase. This can be (mostly) understood on the basis of the corresponding value of $\tilde{\beta}_{\lambda_{L}}$. A large $\tilde{\beta}_{\lambda_{L}}$ is a harbinger of a large scale uncertainty. An inadequate choice of the scale can further exacerbate a large correction driven by $\tilde{\beta}_{\lambda_{L}} \cdot \tilde{\beta}_{\lambda_{L}}$ increases with $\lambda_{2}$ but even for $\lambda_{2} \sim 0, \tilde{\beta}_{\lambda_{L}}$ can be large, leading to a large correction and casting doubt on the perturbative expansion. For example, P3 and most particularly P4, have very large scale uncertainty for all values of $\lambda_{2}$. Based on these oneloop analyses, we make the proposal that such models fail the perturbativity test and should therefore not be considered even though they may pass the tree-level based experimental limits. One should not consider models with $\tilde{\beta}_{\lambda_{L}}$ larger than 20 . For a starter, Table IV shows that when one reaches higher values of $\tilde{\beta}_{\lambda_{L}}$ the corrections reach more than $100 \%$ unless one has carefully picked up an optimal scale. In any case, for large values of $\tilde{\beta}_{\lambda_{L}}$, one is subject to violent variations even if one moves slightly away 
TABLE IV. Tree-level cross section and one-loop correction, $\delta \sigma\left(\bar{\mu}_{\text {dim }}, \lambda_{2}\right)$ for different values of $\lambda_{2}$ and for different renormalization scales, $\bar{\mu}_{\text {dim }}$, for $\lambda_{L}$, for $X X \rightarrow Z Z$, and $v=0.4(\sqrt{s}=204.124)$. The percentage change of the one-loop correction is also given in parenthesis unless the value is higher than $100 \%$ in which case the result is highlighted in bold as a warning for a breakdown of one-loop perturbativity. The loop results (from the numerical evaluation of the automated calculations) are given with sufficient accuracy in order to compare the results against those of the analytical computation given in Eq. (7.3). The first entry in the second column is the tree-level cross section while the second entry in the second column corresponds to $\Delta=\sigma_{\text {tree }}\left(\alpha^{-1}\left(M_{Z}^{2}\right)=128.907\right)-\sigma_{\text {tree }}\left(\alpha^{-1}(0)=137.036\right)$, the "correction" that corresponds to using $\alpha\left(M_{Z}^{2}\right)$ instead of $\alpha$ in the Thomson limit (tree level). The value of $\Delta$ needs to be compared to the result of the full one-loop calculation. The first column gives the parameters of the model, with $M_{X}=100$ for all points, and the corresponding $\lambda_{3,4,5}$. In the same column, we also give $\beta_{\lambda_{L}}$ and a fit of the tree-level cross section as a function of $\lambda_{L}$ (see text). Cross sections are for $\sigma v$ in units of $10^{-26} \mathrm{~cm}^{3} \mathrm{~s}^{-1}$.

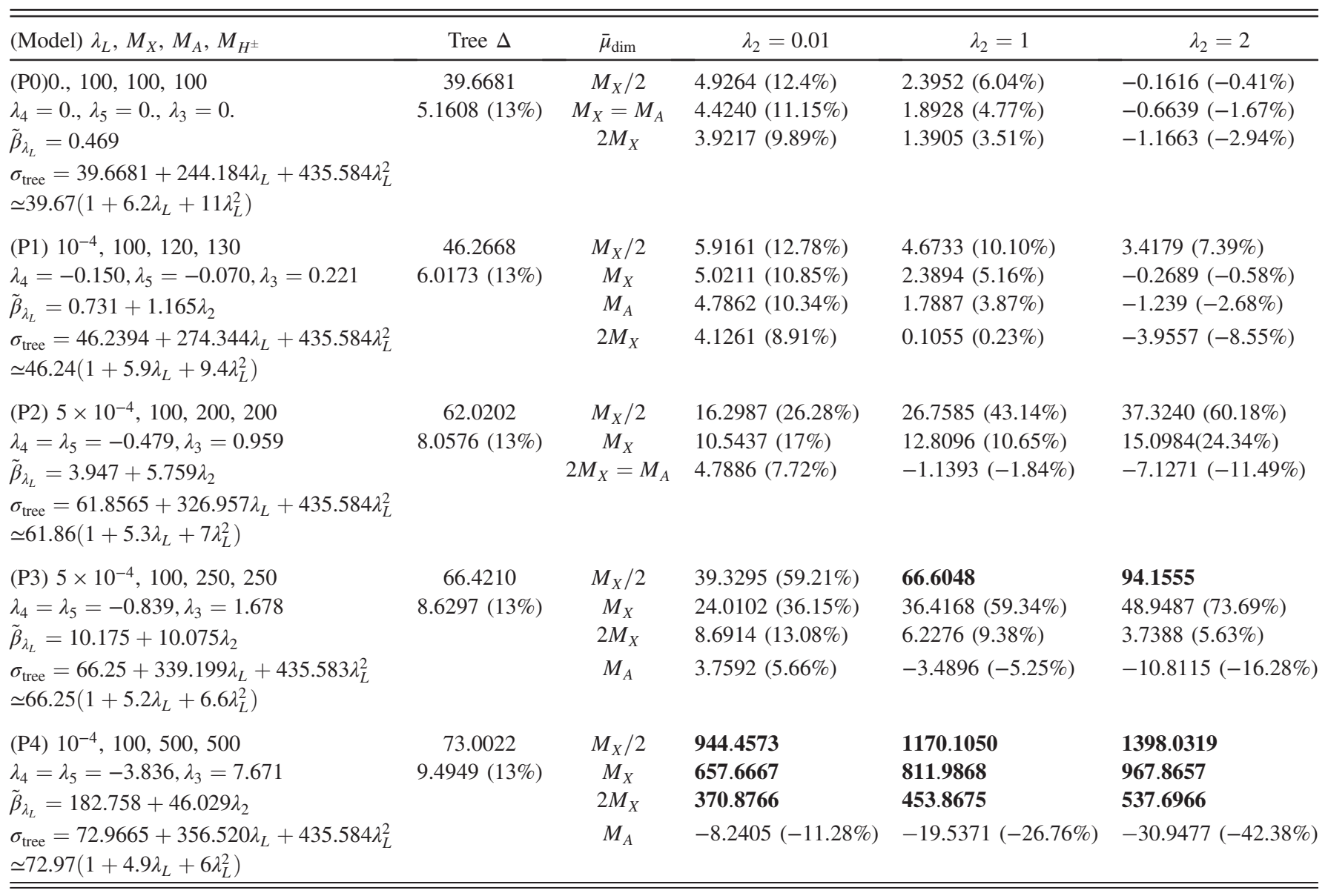

from the optimum scale. Second, if one takes QCD as example with $g_{s}^{2}=4 \pi \alpha_{s}$ one has (in our notation), $\beta_{g_{s}} \sim-13$ while (for the most relevant quantity), $\beta_{\alpha_{s}}=-2.5$ at energy scales where $\alpha_{s}$ is perturbative $\left(\alpha_{s} \sim 0.12\right)$. For $Q^{2} \sim(1 \mathrm{GeV})^{2}$ when $\mathrm{QCD}$ is nonperturbative, $\beta_{\alpha_{s}} \sim 20$. Therefore, $\tilde{\beta}_{\lambda_{L}} \sim 20$ should be a good indicator for the onset of the nonperturbative regime. This indicator when translated in terms of the underlying parameters of the IDM suggests that we should not consider IDM benchmarks points to be theoretically valid if $M_{A}\left(M_{H^{ \pm}}\right)>$ $M_{X}+150 \mathrm{GeV}$; otherwise, the corresponding $\lambda_{3,4,5}$ correspond to values that lead to $\tilde{\beta}_{\lambda_{L}}$ in excess of 20 . This requirement of one-loop perturbativity reduces the IDM parameter space considerably. We will take this conclusion into account to reduce the possibilities for the benchmark points of Table II. We consider the benchmark points $\mathrm{B}, \mathrm{C}, \mathrm{D}, \mathrm{H}$ to be theoretically unreliable.

(iv) In our study of the scale dependence for the partial width $\Gamma_{h \rightarrow X X}$ in Sec. III, we advocated the use of $\mu=\operatorname{Max}\left(2 M_{X}, M_{A}\right)$ as the optimum scale where the radiative corrections were minimized. Table IV suggests a very similar behavior. For the annihilation processes, the typical energy scale of the problem set by kinematics is $\sqrt{s} \simeq 2 M_{X}$, while the typical internal mass is $M_{A}$. Although we are dealing with a process involving four-point functions, the arguments put forward in the case $h \rightarrow X X$ remain the same especially that the scale dependence is embedded in two- and three-point functions. We find 
that $\mu=\operatorname{Max}\left(2 M_{X}, M_{A}\right)$ is once again the best scale. Scales much smaller or larger than this choice lead to very large corrections. This is most flagrant for test points P3 and P4 that we dismissed on the ground of perturbativity, to the point that following this prescription would lead to acceptable corrections if we choose $\mu=M_{A}$. For the "perturbative" test points $\mathrm{P} 0, \mathrm{P} 1$, and $\mathrm{P} 2$, the prescription is most evident for P2. The indication from P1 with $\lambda_{2}=2$ is misleading although the corrections are small for all scales $\left(\tilde{\beta}_{\lambda_{L}}\right.$ is small here), $\mu=2 M_{X}$ does not give the smallest correction because of the $\lambda_{2}$ contribution not originating from $\tilde{\beta}_{\lambda_{L}}$. For $\lambda_{2}=0.01, \mu=2 M_{X}$ gives the smallest correction. These observations will be confirmed also when we study $X X \rightarrow Z f \bar{f}$ and $X X \rightarrow W f \bar{f}^{\prime}[112]$.

We also find that the $\lambda_{L}$ dependence of the $X X \rightarrow$ $W^{+} W^{-}$is practically the same as the $X X \rightarrow Z Z$ cross section. $X X \rightarrow W^{+} W^{-}$will therefore exhibit the same scale dependence as we will see next.

\section{C. $X X \rightarrow Z Z$ and $X X \rightarrow W^{+} W^{-}$at one loop as a function of the relative velocity}

The particular study at $v=0.4$ summarizes in fact the analyses for the whole range of $v$. We therefore briefly discuss here the results for point 1 for the annihilations into both $Z Z$ and $W W$.

Recall that P1 has a small $\tilde{\beta}_{\lambda_{L}}$, and therefore, the scale variation is modest not only for $v=0.4$ as we have discussed but is expected to hold for other values of $v$. Indeed, Figs. 5 show that the radiative corrections, for the range of $v$ relevant for the calculation of the relic density, are within a couple of percent of the results obtained with $v=0.4$, for all cases of the scale $\mu$ and the parameter $\lambda_{2}$. The radiative corrections increase slightly, within a $2 \%$ margin, with the relative velocity, the increase affects $Z Z$ more than $W^{+} W^{-}$as $v$ increases and is due to the fact that $Z Z$ is more sensitive to the threshold for $Z Z$ production for $M_{X}=100 \mathrm{GeV}$. Otherwise, observe that, as expected, in terms of relative corrections, the results are practically the same for both $Z Z$ and $W^{+} W^{-}$production when the same configuration of the scale and $\lambda_{2}$ is taken. More importantly, what the full one-loop correction teaches us is that the "improved" tree-level cross section, with the use of $\alpha\left(M_{Z}^{2}\right)$, as assumed by default in micrOMEGAs, is a good approximation only for vanishingly small $\lambda_{2}$. This "improved" cross section can not, by construction, catch the genuine one-loop effects from the full $\lambda_{2}$ dependence. The prediction of the $\alpha\left(M_{Z}^{2}\right)$ approximation can be quite far from the full one-loop correction for large values of $\lambda_{2}$. For instance, for $\mu=2 M_{X}$ and $\lambda_{2}=2$, the $\alpha\left(M_{Z}^{2}\right)$ cross sections are practically $20 \%$ higher than the full one-loop correction. In general, we see that measured from the treelevel cross section, the corrections decrease as $\lambda_{2}$ increases,
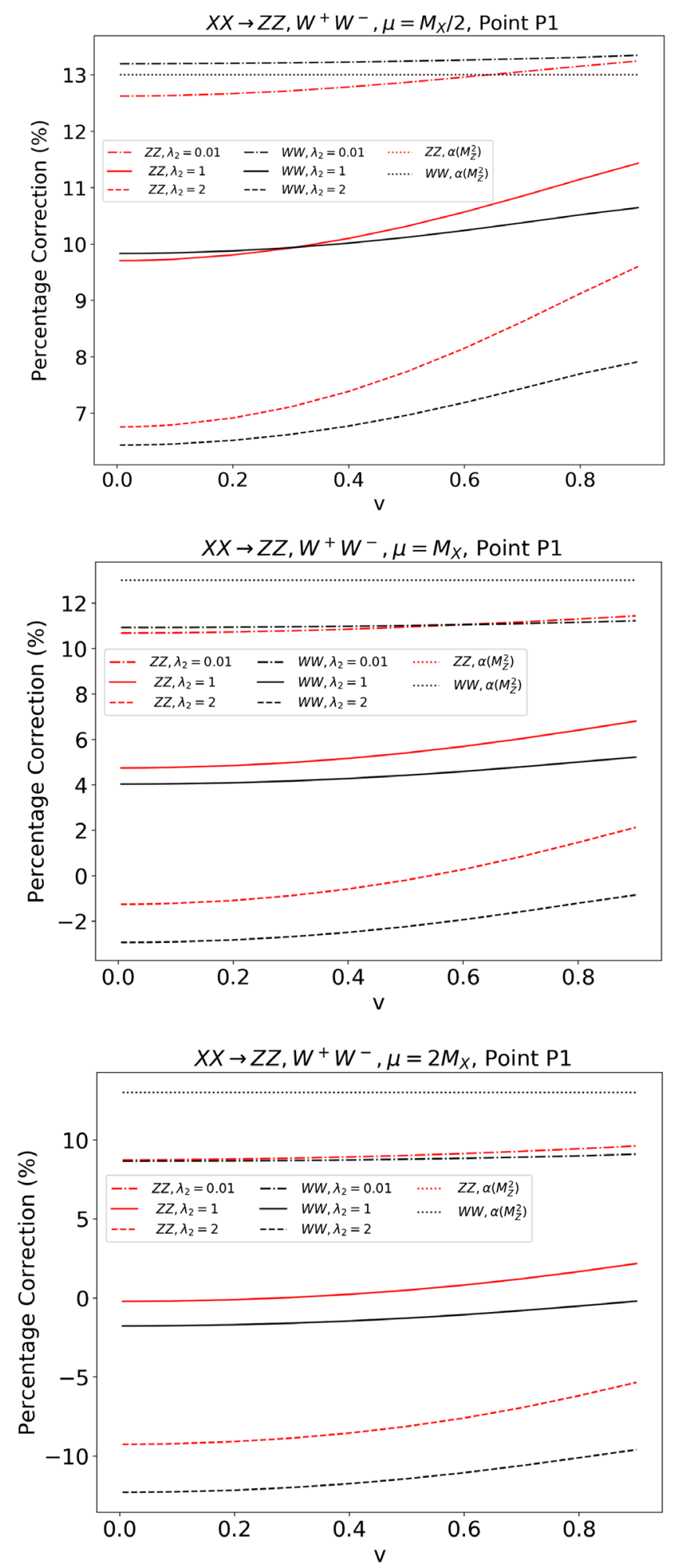

FIG. 5. The relative (to the tree level) full one-loop corrections to the cross sections $X X \rightarrow W^{+} W^{-}, Z Z$ as a function of the relative velocity for test point P1 with $\mu=M_{X} / 2, M_{X}, 2 M_{X}$ (from left to right). Three values of $\lambda_{2}$ are considered. The percentage deviation due to choosing as input $\alpha\left(M_{Z}^{2}\right)$ instead of $\alpha(0)$ is also shown. This correction $(\sim 13 \%)$ is the same for both $Z Z$ and $W W$. It is therefore superimposed for the two cross sections and appears as a common line. 
and they also decrease as the scale increases, a behavior that is fully explained by our $\lambda_{L}, \tilde{\beta}_{\lambda_{L}}$ discussion exposed through Eq. (7.4). Therefore, we recommend for such scenarios, with small $\tilde{\beta}_{\lambda_{L}}$, to apply a theoretical uncertainty to the prediction of micrOMEGAs ranging from $-20 \%$ to $-4 \%$ to account for the scale uncertainty and the $\lambda_{2}$ dependence that a tree-level calculation is not sensitive to.

\section{CONCLUSIONS}

Compared to a much studied model like the MSSM, the IDM with the addition of one scalar doublet to the SM is quite simple and economical in terms of the number of parameters it involves. Yet, it has a rich structure and constitutes an excellent laboratory to address many technical issues about renormalization of a BSM model. Namely, what physical input parameters could one define to perform a full one-loop calculation. Using the masses of the new fields (particles) of the model seem a most unambiguous scheme to define the model. However, even if all masses are given, we still need two more parameters, $\lambda_{L}$ (or some other combination of the underlying parameters of the Lagrangian) and $\lambda_{2}$ to compute observables, most importantly, cross sections. $\lambda_{L}$, which measures the strength of the coupling of the SM Higgs boson to the DM particle, is needed for the (tree-level) calculation of the annihilation of DM to SM particles, besides the gauge couplings that we define from SM observables. The quartic coupling $\lambda_{2}$ represents interactions solely within the dark sector. Nonetheless, loop effects introduce an important $\lambda_{2}$ dependence of the annihilation cross sections. This indirect one-loop effect therefore introduces an uncertainty in the prediction of an observable such as the relic density. $\lambda_{L}$ also introduces an uncertainty of a different sort. Either this coupling is extracted from the partial invisible width of the Higgs boson, phase space allowing, or as we advocated in this paper, an $\overline{\mathrm{MS}}$ scheme is prescribed to allow a full oneloop calculation. The latter introduces a renormalization scale dependence in the one-loop predictions. If the $\overline{\mathrm{MS}}$ prescription is applied to $\lambda_{L}$ only, we have shown how the scale dependence can be tracked through the $\beta$ constant for $\lambda_{L}\left(\tilde{\beta}_{\lambda_{L}}\right)$ and the $\lambda_{L}$ parametrization of the tree-level observable. We have also argued how to reduce the scale uncertainty by choosing an optimal scale which we conjecture to be close to the largest scale involved in the process. The overall theoretical uncertainty should be estimated by varying the scale around this optimal value and also by varying $\lambda_{2}$, a quantity the annihilation of DM to SM particles does not depend on. The study of the theoretical uncertainty at one-loop has also led us to introduce a new criterion for the perturbativity requirement: only configurations with small enough $\tilde{\beta}_{\lambda_{L}}$ qualify. An important consequence from this requirement is that one should restrict the study of the IDM to the range
$M_{A}, \sim M_{H}^{ \pm}<M_{X}+150 \mathrm{GeV}$. From the point of view of the relic density calculation, the IDM, even in the narrow range of low masses $\left(55<M_{X}<75 \mathrm{GeV}\right)$, involves the main known mechanisms in the freeze-out scenario: annihilation in the continuum, coannihilation, annihilation through a resonance. We have conducted a very thorough investigation on the allowed parameter space of the model, which includes new LHC and direct detection data to delimit the range of the low mass IDM scenarios and the DM annihilation mechanisms that are involved. A set of representative IDM points covering these mechanisms was presented. It will serve as a starting point to conduct full one-loop calculations in these three scenarios. $X X \rightarrow W f \bar{f}^{\prime}$ (a $2 \rightarrow 3$ process) at one-loop, which has never been attended before, will figure in all three scenarios but with varying degree of importance. Salient features of this process are contained in the $2 \rightarrow 2$ processes $X X \rightarrow W^{+} W^{-}, Z Z$, which we studied in this paper. Crucial technical process specific issues, beyond the general renormalization procedure presented at some length here, are presented in the accompanying papers that cover each of the three scenarios. The calculation of the relic density at one-loop when the mechanism is dominated by coannihilation will be presented in Ref. [111]. If we set aside the induced subdominant production through $X X \rightarrow W f \bar{f}^{\prime}$ (a $2 \rightarrow 3$ process), the dominant mechanism is $A X$ coannihilation to a fermion pair. In Ref. [112], we consider three benchmark points to present results for $X X \rightarrow W f \bar{f}^{\prime}, X X \rightarrow Z f \bar{f}$ and how the corrected cross sections translate into the calculation of the relic density. Finally in Ref. [110], the resonance region, we show how to extend the OS scheme that we detailed in the present paper by supplementing a complex scheme that avoids the issue of double counting in the presence of a width. The width, necessary in the tree-level calculation, is in fact induced at the loop level calculation since the width represents the imaginary part of the self-energy contribution. Again, the IDM can be a good example which illustrates how a loop calculation for a process that proceeds through a resonance should be conducted.

\section{ACKNOWLEDGMENTS}

We thank Alexander Pukhov for helpful discussions. H.S. is supported by the National Natural Science Foundation of China (Grants No. 12075043 and No. 11675033). He warmly thanks the CPTGA and LAPTh for support during his visit to France when this work was initiated. S. B. is grateful for the support received from IPPP, Durham, UK, where most of this work was performed and is also grateful to the support received from LAPTh where this work was initiated. N. C. is financially supported by IISc (Indian Institute of Science, Bangalore, India) through the C. V. Raman postdoctoral fellowship. $\mathrm{He}$ also acknowledges the support received from DST, India, under grant number IFA19-PH237 (INSPIRE Faculty Award). 
[1] P. A. R. Ade et al. (Planck Collaboration), Planck 2015 results. XIII. Cosmological parameters, Astron. Astrophys. 594, A13 (2016).

[2] F. Boudjema, A. Semenov, and D. Temes, Self-annihilation of the neutralino dark matter into two photons or a $z$ and a photon in the minimal supersymmetric standard model, Phys. Rev. D 72, 055024 (2005).

[3] N. Baro, F. Boudjema, and A. Semenov, Full one-loop corrections to the relic density in the MSSM: A few examples, Phys. Lett. B 660, 550 (2008),

[4] N. Baro, F. Boudjema, and A. Semenov, Automatised full one-loop renormalisation of the MSSM. I. The Higgs sector, the issue of $\tan$ (beta) and gauge invariance, Phys. Rev. D 78, 115003 (2008).

[5] N. Baro, F. Boudjema, G. Chalons, and S. Hao, Relic density at one-loop with gauge boson pair production, Phys. Rev. D 81, 015005 (2010).

[6] F. Boudjema, G. Drieu La Rochelle, and S. Kulkarni, Oneloop corrections, uncertainties and approximations in neutralino annihilations: Examples, Phys. Rev. D 84, 116001 (2011).

[7] A. Chatterjee, M. Drees, and S. Kulkarni, Radiative corrections to the neutralino dark matter relic densityan effective coupling approach, Phys. Rev. D 86, 105025 (2012).

[8] F. Boudjema, G. Drieu La Rochelle, and A. Mariano, Relic density calculations beyond tree-level, exact calculations versus effective couplings: The ZZ final state, Phys. Rev. D 89, 115020 (2014).

[9] F. Bojarski, G. Chalons, D. Lopez-Val, and T. Robens, Heavy to light Higgs boson decays at NLO in the singlet extension of the standard model, J. High Energy Phys. 02 (2016) 147.

[10] S. Banerjee, F. Boudjema, N. Chakrabarty, G. Chalons, and H. Sun, Relic density of dark matter in the inert doublet model beyond leading order: The heavy mass case, Phys. Rev. D 100, 095024 (2019).

[11] B. Herrmann, M. Klasen, and K. Kovarik, Neutralino annihilation into massive quarks with SUSY-QCD corrections, Phys. Rev. D 79, 061701 (2009).

[12] B. Herrmann, M. Klasen, and K. Kovarik, SUSY-QCD effects on neutralino dark matter annihilation beyond scalar or gaugino mass unification, Phys. Rev. D 80, 085025 (2009).

[13] J. Harz, B. Herrmann, M. Klasen, K. Kovarik, and Q. L. Boulc'h, Neutralino-stop coannihilation into electroweak gauge and Higgs bosons at one loop, Phys. Rev. D 87, 054031 (2013).

[14] B. Herrmann, M. Klasen, K. Kovarik, M. Meinecke, and P. Steppeler, One-loop corrections to gaugino (co)annihilation into quarks in the MSSM, Phys. Rev. D 89, 114012 (2014).

[15] J. Harz, B. Herrmann, M. Klasen, and K. Kovarik, Oneloop corrections to neutralino-stop coannihilation revisited, Phys. Rev. D 91, 034028 (2015).

[16] J. Harz, B. Herrmann, M. Klasen, K. Kovařík, and M. Meinecke, SUSY-QCD corrections to stop annihilation into electroweak final states including coulomb enhancement effects, Phys. Rev. D 91, 034012 (2015).
[17] J. Branahl, J. Harz, B. Herrmann, M. Klasen, K. Kovařík, and S. Schmiemann, SUSY-QCD corrected and Sommerfeld enhanced stau annihilation into heavy quarks with scheme and scale uncertainties, Phys. Rev. D 100, 115003 (2019).

[18] J. Hisano, S. Matsumoto, and M. M. Nojiri, Unitarity and higher order corrections in neutralino dark matter annihilation into two photons, Phys. Rev. D 67, 075014 (2003).

[19] J. Hisano, S. Matsumoto, and M. M. Nojiri, Explosive Dark Matter Annihilation, Phys. Rev. Lett. 92, 031303 (2004).

[20] J. Hisano, S. Matsumoto, M. M. Nojiri, and O. Saito, Nonperturbative effect on dark matter annihilation and gamma ray signature from galactic center, Phys. Rev. D 71, 063528 (2005).

[21] M. Cirelli, A. Strumia, and M. Tamburini, Cosmology and astrophysics of minimal dark matter, Nucl. Phys. B787, 152 (2007).

[22] N. Arkani-Hamed, D. P. Finkbeiner, T. R. Slatyer, and N. Weiner, A theory of dark matter, Phys. Rev. D 79, 015014 (2009).

[23] T. Hambye, F. S. Ling, L. Lopez Honorez, and J. Rocher, Scalar multiplet dark matter, J. High Energy Phys. 07 (2009) 090.

[24] M. Drees, J. M. Kim, and K. I. Nagao, Potentially large one-loop corrections to WIMP annihilation, Phys. Rev. D 81, 105004 (2010).

[25] A. Hryczuk and R. Iengo, The one-loop and Sommerfeld electroweak corrections to the Wino dark matter annihilation, J. High Energy Phys. 01 (2012) 163.

[26] M. Beneke, C. Hellmann, and P. Ruiz-Femenia, Heavy neutralino relic abundance with Sommerfeld enhancements - a study of pMSSM scenarios, J. High Energy Phys. 03 (2015) 162.

[27] K. Blum, R. Sato, and T. R. Slatyer, Self-consistent calculation of the Sommerfeld enhancement, J. Cosmol. Astropart. Phys. 06 (2016) 021.

[28] A. Hryczuk, The Sommerfeld enhancement for scalar particles and application to sfermion co-annihilation regions, Phys. Lett. B 699, 271 (2011).

[29] S. Biondini and M. Laine, Re-derived overclosure bound for the inert doublet model, J. High Energy Phys. 08 (2017) 047.

[30] P. Ciafaloni, M. Cirelli, D. Comelli, A. De Simone, A. Riotto, and A. Urbano, On the importance of electroweak corrections for majorana dark matter indirect detection, J. Cosmol. Astropart. Phys. 06 (2011) 018.

[31] P. Ciafaloni, D. Comelli, A. De Simone, A. Riotto, and A. Urbano, Electroweak bremsstrahlung for wino-like dark matter annihilations, J. Cosmol. Astropart. Phys. 06 (2012) 016.

[32] G. Ovanesyan, N. L. Rodd, T. R. Slatyer, and I. W. Stewart, One-loop correction to heavy dark matter annihilation, Phys. Rev. D 95, 055001 (2017).

[33] P. Ruiz-Femenía, Sommerfeld enhancements and relic abundance of neutralino dark matter in the general MSSM, Nucl. Part. Phys. Proc. 273-275, 425 (2016).

[34] G. Chalons, M. J. Dolan, and C. McCabe, Neutralino dark matter and the Fermi gamma-ray lines, J. Cosmol. Astropart. Phys. 02 (2013) 016. 
[35] M. Baumgart, T. Cohen, I. Moult, N. L. Rodd, T. R. Slatyer, M. P. Solon, I. W. Stewart, and V. Vaidya, Resummed photon spectra for WIMP annihilation, J. High Energy Phys. 03 (2018) 117.

[36] M. Beneke, A. Broggio, C. Hasner, and M. Vollmann, Energetic $\gamma$-rays from $\mathrm{TeV}$ scale dark matter annihilation resummed, Phys. Lett. B 786, 347 (2018).

[37] M. Baumgart, T. Cohen, E. Moulin, I. Moult, L. Rinchiuso, N. L. Rodd, T. R. Slatyer, I. W. Stewart, and V. Vaidya, Precision photon spectra for Wino annihilation, J. High Energy Phys. 01 (2019) 036.

[38] M. Beneke, A. Broggio, C. Hasner, K. Urban, and M. Vollmann, Resummed photon spectrum from dark matter annihilation for intermediate and narrow energy resolution, J. High Energy Phys. 08 (2019) 103.

[39] M. Beneke, C. Hasner, K. Urban, and M. Vollmann, Precise yield of high-energy photons from Higgsino dark matter annihilation, J. High Energy Phys. 03 (2020) 030.

[40] L. Rinchiuso, O. Macias, E. Moulin, N. L. Rodd, and T. R. Slatyer, Prospects for detecting heavy WIMP dark matter with the cherenkov telescope array: The Wino and Higgsino, Phys. Rev. D 103, 023011 (2021).

[41] M. Cirelli, N. Fornengo, and A. Strumia, Minimal dark matter, Nucl. Phys. B753, 178 (2006).

[42] J. Hisano, K. Ishiwata, and N. Nagata, A complete calculation for direct detection of Wino dark matter, Phys. Lett. B 690, 311 (2010).

[43] M. Klasen, C. E. Yaguna, and J. D. Ruiz-Alvarez, Electroweak corrections to the direct detection cross section of inert higgs dark matter, Phys. Rev. D 87, 075025 (2013).

[44] U. Haisch and F. Kahlhoefer, On the importance of loopinduced spin-independent interactions for dark matter direct detection, J. Cosmol. Astropart. Phys. 04 (2013) 050 .

[45] J. Hisano, K. Ishiwata, and N. Nagata, QCD effects on direct detection of Wino dark matter, J. High Energy Phys. 06 (2015) 097.

[46] T. Abe and R. Sato, Quantum corrections to the spinindependent cross section of the inert doublet dark matter, J. High Energy Phys. 03 (2015) 109.

[47] J. Herrero-Garcia, E. Molinaro, and M. A. Schmidt, Dark matter direct detection of a fermionic singlet at one loop, Eur. Phys. J. C 78, 471 (2018).

[48] T. Abe, M. Fujiwara, and J. Hisano, Loop corrections to dark matter direct detection in a pseudoscalar mediator dark matter model, J. High Energy Phys. 02 (2019) 028.

[49] T. Han, H. Liu, S. Mukhopadhyay, and X. Wang, Dark matter blind spots at one-loop, J. High Energy Phys. 03 (2019) 080.

[50] S. Glaus, M. Mühlleitner, J. Müller, S. Patel, and R. Santos, Electroweak corrections to dark matter direct detection in a vector dark matter model, J. High Energy Phys. 10 (2019) 152.

[51] C.-T. Lu, V. Q. Tran, and Y.-L. S. Tsai, Confronting dark matter co-annihilation of Inert two Higgs doublet model with a compressed mass spectrum, J. High Energy Phys. 06 (2020) 033.

[52] N. G. Deshpande and E. Ma, Pattern of symmetry breaking with two Higgs doublets, Phys. Rev. D 18, 2574 (1978).
[53] R. Barbieri, L. J. Hall, and V. S. Rychkov, Improved naturalness with a heavy Higgs: An alternative road to LHC physics, Phys. Rev. D 74, 015007 (2006).

[54] T. Hambye and M. H. G. Tytgat, Electroweak symmetry breaking induced by dark matter, Phys. Lett. B 659, 651 (2008).

[55] T. Wizansky, Finite temperature corrections to relic density calculations, Phys. Rev. D 74, 065007 (2006).

[56] M. Beneke, F. Dighera, and A. Hryczuk, Relic density computations at NLO: infrared finiteness and thermal correction, J. High Energy Phys. 10 (2014) 045.

[57] M. Beneke, F. Dighera, and A. Hryczuk, Finite-temperature modification of heavy particle decay and dark matter annihilation, J. High Energy Phys. 09 (2016) 031.

[58] L. Lopez Honorez, E. Nezri, J. F. Oliver, and M. H. G. Tytgat, The inert doublet model: An archetype for dark matter, J. Cosmol. Astropart. Phys. 02 (2007) 028.

[59] Q.-H. Cao, E. Ma, and G. Rajasekaran, Observing the dark scalar doublet and its impact on the standard-model Higgs boson at colliders, Phys. Rev. D 76, 095011 (2007).

[60] P. Agrawal, E. M. Dolle, and C. A. Krenke, Signals of inert doublet dark matter in neutrino telescopes, Phys. Rev. D 79, 015015 (2009).

[61] E. Lundstrom, M. Gustafsson, and J. Edsjo, The inert doublet model and LEP II limits, Phys. Rev. D 79, 035013 (2009).

[62] S. Andreas, M. H. G. Tytgat, and Q. Swillens, Neutrinos from inert doublet dark matter, J. Cosmol. Astropart. Phys. 04 (2009) 004.

[63] C. Arina, F.-S. Ling, and M. H. G. Tytgat, IDM and iDM or The inert doublet model and inelastic dark matter, J. Cosmol. Astropart. Phys. 10 (2009) 018.

[64] E. Dolle, X. Miao, S. Su, and B. Thomas, Dilepton signals in the inert doublet model, Phys. Rev. D 81, 035003 (2010).

[65] E. Nezri, M. H. G. Tytgat, and G. Vertongen, e+ and $\bar{p}$ from inert doublet model dark matter, J. Cosmol. Astropart. Phys. 04 (2009) 014.

[66] X. Miao, S. Su, and B. Thomas, Trilepton signals in the inert doublet model, Phys. Rev. D 82, 035009 (2010).

[67] J.-O. Gong, H. M. Lee, and S. K. Kang, Inflation and dark matter in two Higgs doublet models, J. High Energy Phys. 04 (2012) 128.

[68] M. Gustafsson, S. Rydbeck, L. Lopez-Honorez, and E. Lundstrom, Status of the inert doublet model and the role of multileptons at the LHC, Phys. Rev. D 86, 075019 (2012).

[69] B. Swiezewska and M. Krawczyk, Diphoton rate in the inert doublet model with a $125 \mathrm{GeV}$ Higgs boson, Phys. Rev. D 88, 035019 (2013).

[70] L. Wang and X.-F. Han, LHC diphoton Higgs signal and top quark forward-backward asymmetry in quasi-inert Higgs doublet model, J. High Energy Phys. 05 (2012) 088.

[71] A. Goudelis, B. Herrmann, and O. Stal, Dark matter in the inert doublet model after the discovery of a Higgs-like boson at the LHC, J. High Energy Phys. 09 (2013) 106.

[72] A. Arhrib, Y.-L. S. Tsai, Q. Yuan, and T.-C. Yuan, An updated analysis of inert Higgs doublet model in light of the recent results from LUX, PLANCK, AMS-02 and LHC, J. Cosmol. Astropart. Phys. 06 (2014) 030. 
[73] M. Krawczyk, D. Sokolowska, P. Swaczyna, and B. Swiezewska, Constraining inert dark matter by $R_{\gamma \gamma}$ and WMAP data, J. High Energy Phys. 09 (2013) 055.

[74] P. Osland, A. Pukhov, G. M. Pruna, and M. Purmohammadi, Phenomenology of charged scalars in the $C P$-Violating inert-doublet model, J. High Energy Phys. 04 (2013) 040.

[75] A. Arhrib, R. Benbrik, J. El Falaki, and A. Jueid, Radiative corrections to the triple Higgs coupling in the inert Higgs doublet model, J. High Energy Phys. 12 (2015) 007.

[76] N. Blinov, J. Kozaczuk, D. E. Morrissey, and A. de la Puente, Compressing the inert doublet model, Phys. Rev. D 93, 035020 (2016).

[77] M. A. Díaz, B. Koch, and S. Urrutia-Quiroga, Constraints to dark matter from inert Higgs doublet model, Adv. High Energy Phys. 2016, 8278375 (2016).

[78] A. Ilnicka, M. Krawczyk, and T. Robens, Inert doublet model in light of LHC Run I and astrophysical data, Phys. Rev. D 93, 055026 (2016).

[79] G. Belanger, B. Dumont, A. Goudelis, B. Herrmann, S. Kraml, and D. Sengupta, Dilepton constraints in the inert doublet model from Run1 of the LHC, Phys. Rev. D 91, 115011 (2015).

[80] A. Carmona and M. Chala, Composite dark sectors, J. High Energy Phys. 06 (2015) 105.

[81] S. Kanemura, M. Kikuchi, and K. Sakurai, Testing the dark matter scenario in the inert doublet model by future precision measurements of the Higgs boson couplings, Phys. Rev. D 94, 115011 (2016).

[82] F. S. Queiroz and C. E. Yaguna, The CTA aims at the inert doublet model, J. Cosmol. Astropart. Phys. 02 (2016) 038.

[83] A. Belyaev, G. Cacciapaglia, I. P. Ivanov, F. Rojas-Abatte, and M. Thomas, Anatomy of the inert two higgs doublet model in the light of the LHC and non-LHC dark matter searches, Phys. Rev. D 97, 035011 (2018).

[84] G. Arcadi, A. Djouadi, and M. Raidal, Dark Matter through the Higgs portal, Phys. Rep. 842, 1 (2020).

[85] B. Eiteneuer, A. Goudelis, and J. Heisig, The inert doublet model in the light of Fermi-LAT gamma-ray data: A global fit analysis, Eur. Phys. J. C 77, 624 (2017).

[86] A. Ilnicka, T. Robens, and T. Stefaniak, Constraining extended scalar sectors at the LHC and beyond, Mod. Phys. Lett. A 33, 1830007 (2018).

[87] J. Kalinowski, W. Kotlarski, T. Robens, D. Sokolowska, and A. F. Zarnecki, Benchmarking the inert doublet model for $e^{+} e^{-}$colliders, J. High Energy Phys. 12 (2018) 081.

[88] A. Bhardwaj, P. Konar, T. Mandal, and S. Sadhukhan, Probing the inert doublet model using jet substructure with a multivariate analysis, Phys. Rev. D 100, 055040 (2019).

[89] R. Basu, S. Banerjee, M. Pandey, and D. Majumdar, Lower bounds on dark matter annihilation cross-sections by studying the fluctuations of $21-\mathrm{cm}$ line with dark matter candidate in inert doublet model (IDM) with the combined effects of dark matter scattering and annihilation, arXiv: 2010.11007.

[90] A. Jueid, J. Kim, S. Lee, S. Y. Shim, and J. Song, Phenomenology of the inert doublet model with a global U(1) symmetry, Phys. Rev. D 102, 075011 (2020).

[91] N. Blinov, S. Profumo, and T. Stefaniak, The electroweak phase transition in the inert doublet model, J. Cosmol. Astropart. Phys. 07 (2015) 028.
[92] G. Gil, P. Chankowski, and M. Krawczyk, Inert dark matter and strong electroweak phase transition, Phys. Lett. B 717, 396 (2012).

[93] S. Fabian, F. Goertz, and Y. Jiang, Dark matter and nature of electroweak phase transition with an inert doublet, arXiv:2012.12847.

[94] P. M. Ferreira and D. R. T. Jones, Bounds on scalar masses in two Higgs doublet models, J. High Energy Phys. 08 (2009) 069.

[95] B. Świeżewska, Yukawa independent constraints for twoHiggs-doublet models with a $125 \mathrm{GeV}$ Higgs boson, Phys. Rev. D 88, 055027 (2013).

[96] P. M. Ferreira and B. Swiezewska, One-loop contributions to neutral minima in the inert doublet model, J. High Energy Phys. 04 (2016) 099.

[97] B. Swiezewska, Inert scalars and vacuum metastability around the electroweak scale, J. High Energy Phys. 07 (2015) 118.

[98] N. Khan and S. Rakshit, Constraints on inert dark matter from the metastability of the electroweak vacuum, Phys. Rev. D 92, 055006 (2015).

[99] S. Kanemura, S. Kiyoura, Y. Okada, E. Senaha, and C. P. Yuan, New physics effect on the Higgs selfcoupling, Phys. Lett. B 558, 157 (2003).

[100] E. Senaha, Radiative corrections to triple Higgs coupling and electroweak phase transition: Beyond one-loop analysis, Phys. Rev. D 100, 055034 (2019).

[101] J. Braathen and S. Kanemura, On two-loop corrections to the Higgs trilinear coupling in models with extended scalar sectors, Phys. Lett. B 796, 38 (2019).

[102] H. Abouabid, A. Arhrib, R. Benbrik, J. E. Falaki, B. Gong, W. Xie, and Q.-S. Yan, One-loop radiative corrections to $e^{+} e^{-} \rightarrow Z h^{0} / H^{0} A^{0}$ in the inert Higgs doublet model, J. High Energy Phys. 05 (2021) 100.

[103] A. Arhrib, R. Benbrik, and N. Gaur, $H \rightarrow \gamma \gamma$ in Inert Higgs Doublet Model, Phys. Rev. D 85, 095021 (2012).

[104] M. Gustafsson, E. Lundstrom, L. Bergstrom, and J. Edsjo, Significant Gamma Lines from Inert Higgs Dark Matter, Phys. Rev. Lett. 99, 041301 (2007).

[105] C. Garcia-Cely, M. Gustafsson, and A. Ibarra, Probing the inert doublet dark matter model with cherenkov telescopes, J. Cosmol. Astropart. Phys. 02 (2016) 043.

[106] S. Banerjee and N. Chakrabarty, A revisit to scalar dark matter with radiative corrections, J. High Energy Phys. 05 (2019) 150.

[107] G. Belanger, F. Boudjema, A. Pukhov, and A. Semenov, micrOMEGAs_3: A program for calculating dark matter observables, Comput. Phys. Commun. 185, 960 (2014).

[108] B. C. Allanach, G. Belanger, F. Boudjema, and A. Pukhov, Requirements on collider data to match the precision of wmap on supersymmetric dark matter, J. High Energy Phys. 12 (2004) 020.

[109] F. Boudjema and E. Chopin, Double Higgs production at the linear colliders and the probing of the Higgs selfcoupling, Z. Phys. C 73, 85 (1996).

[110] S. Banerjee, F. Boudjema, N. Chakrabarty, and H. Sun, this issue, Relic density of dark matter in the inert doublet model beyond leading order for the low mass region: 4. The Higgs resonance region, Phys. Rev. D 104, 075005 (2021). 
[111] S. Banerjee, F. Boudjema, N. Chakrabarty, and H. Sun, following paper, Relic density of dark matter in the inert doublet model beyond leading order for the low mass region: 2. Co-annihilation, Phys. Rev. D 104, 075003 (2021).

[112] S. Banerjee, F. Boudjema, N. Chakrabarty, and H. Sun, this issue, Relic density of dark matter in the inert doublet model beyond leading order for the low mass region: 3 . Annihilation in 3-body final state, Phys. Rev. D 104, 075004 (2021).

[113] G. Bélanger, V. Bizouard, F. Boudjema, and G. Chalons, One-loop renormalization of the NMSSM in SloopS: The neutralino-chargino and sfermion sectors, Phys. Rev. D 93, 115031 (2016).

[114] G. Bélanger, V. Bizouard, F. Boudjema, and G. Chalons, One-loop renormalization of the NMSSM in SloopS. II. The Higgs sector, Phys. Rev. D 96, 015040 (2017).

[115] T. Hahn, Generating Feynman diagrams and amplitudes with FeynArts 3, Comput. Phys. Commun. 140, 418 (2001).

[116] T. Hahn, S. Passehr, and C. Schappacher, FormCalc 9 and extensions, J. Phys. Conf. Ser. 762, 012065 (2016).

[117] T. Hahn and M. Perez-Victoria, Automatized one loop calculations in four-dimensions and D-dimensions, Comput. Phys. Commun. 118, 153 (1999).

[118] A. Semenov, LanHEP: A Package for the automatic generation of Feynman rules in field theory. Version 3.0, Comput. Phys. Commun. 180, 431 (2009).

[119] A. Semenov, LanHEP-A package for automatic generation of Feynman rules from the Lagrangian. Version 3.2, Comput. Phys. Commun. 201, 167 (2016).

[120] G. Belanger, F. Boudjema, A. Pukhov, and A. Semenov, micromEGAs: A Program for calculating the relic density in the MSSM, Comput. Phys. Commun. 149, 103 (2002).

[121] G. Belanger, F. Boudjema, A. Pukhov, and A. Semenov, micrOMEGAs 2.0: A Program to calculate the relic density of dark matter in a generic model, Comput. Phys. Commun. 176, 367 (2007).

[122] G. Belanger, F. Boudjema, A. Goudelis, A. Pukhov, and B. Zaldivar, micromegas5.0: Freeze-in, Comput. Phys. Commun. 231, 173 (2018).

[123] H. E. Haber and R. Hempfling, The renormalization group improved Higgs sector of the minimal supersymmetric model, Phys. Rev. D 48, 4280 (1993).

[124] J. Kalinowski, T. Robens, D. Sokolowska, and A. F. Zarnecki, IDM benchmarks for the LHC and future colliders, arXiv:2012.14818.

[125] E. Aprile et al. (XENON Collaboration), Dark Matter Search Results from a One Ton-Year Exposure of XENON1T, Phys. Rev. Lett. 121, 111302 (2018).

[126] M. Aaboud et al. (ATLAS Collaboration), Constraints on off-shell Higgs boson production and the Higgs boson total width in $Z Z \rightarrow 4 \ell$ and $Z Z \rightarrow 2 \ell 2 \nu$ final states with the ATLAS detector, Phys. Lett. B 786, 223 (2018).

[127] A. M. Sirunyan et al. (CMS Collaboration), Measurements of the Higgs boson width and anomalous $H V V$ couplings from on-shell and off-shell production in the four-lepton final state, Phys. Rev. D 99, 112003 (2019).

[128] S. Kraml, T. Q. Loc, D. T. Nhung, and L. D. Ninh, Constraining new physics from Higgs measurements with Lilith: Update to LHC Run 2 results, SciPost Phys. 7, 052 (2019).

[129] P. D. Group, Review of particle physics, Prog. Theor. Exp. Phys. 2020, 083 C01 (2020); See in particular, pages 13 and 14 online http://pdg.lbl.gov/2019/reviews/rpp2019rev-higgs-boson.pdf.

[130] D. de Florian et al. (LHC Higgs Cross Section Working Group Collaboration), Handbook of LHC Higgs cross sections: 4. Deciphering the nature of the higgs sector, arXiv:1610.07922.

[131] M.E. Peskin and T. Takeuchi, Estimation of oblique electroweak corrections, Phys. Rev. D 46, 381 (1992).

[132] M. Baak, M. Goebel, J. Haller, A. Hoecker, D. Kennedy, K. Mönig et al., Updated status of the global electroweak fit and constraints on new physics, Eur. Phys. J. C 72 (2012).

[133] A. Pierce and J. Thaler, Natural dark matter from an unnatural Higgs boson and new colored particles at the TeV scale, J. High Energy Phys. 08 (2007) 026.

[134] A. Datta, N. Ganguly, N. Khan, and S. Rakshit, Exploring collider signatures of the inert Higgs doublet model, Phys. Rev. D 95, 015017 (2017).

[135] E. Dolle, X. Miao, S. Su, and B. Thomas, Dilepton signals in the inert doublet model, Phys. Rev. D 81, 035003 (2010).

[136] LHC SUSY cross section working group, https://twiki.cern .ch/twiki/bin/view/lhcphysics/susycrosssections.

[137] B. Dumont, B. Fuks, S. Kraml, S. Bein, G. Chalons, E. Conte, S. Kulkarni, D. Sengupta, and C. Wymant, Toward a public analysis database for LHC new physics searches using MadAnalysis 5, Eur. Phys. J. C 75, 56 (2015).

[138] E. Conte and B. Fuks, Confronting new physics theories to LHC data with MadAnalysis 5, Int. J. Mod. Phys. A 33, 1830027 (2018).

[139] J. Y. Araz, B. Fuks, and G. Polykratis, Simplified fast detector simulation in MadAnalysis 5, Eur. Phys. J. C 81, 329 (2021).

[140] G. Aad et al. (ATLAS Collaboration), Search for electroweak production of charginos and sleptons decaying into final states with two leptons and missing transverse momentum in $\sqrt{s}=13 \mathrm{TeV} p p$ collisions using the ATLAS detector, Eur. Phys. J. C 80, 123 (2020).

[141] B. Fuks and J. Y. Araz, Re-implementation of a search for sleptons and electroweakinos in the dilepton + MET channel $139 \mathrm{fb}^{-1}$, Report No. ATLAS-SUSY-2018-32.

[142] A. Sirunyan et al. (CMS Collaboration), Search for electroweak production of charginos and neutralinos in multilepton final states in proton-proton collisions at $\sqrt{s}=13$ TeV, J. High Energy Phys. 03 (2018) 166.

[143] B. Fuks and S. Mondal, MadAnalysis 5 implementation of the CMS search for supersymmetry in the multilepton channel with $35.9 \mathrm{fb}^{-1}$ of $13 \mathrm{TeV}$ LHC data, Report No. CMS-SUS-16-039.

[144] A. M. Sirunyan et al. (CMS Collaboration), Search for dark matter and unparticles in events with a $\mathrm{Z}$ boson and missing transverse momentum in proton-proton collisions at $\sqrt{s}=13 \mathrm{TeV}$, J. High Energy Phys. 03 (2017) 061.

[145] B. Fuks, MadAnalysis 5 implementation of the mono-Z analysis of CMS with $2.3 \mathrm{fb}^{-1}$ of data, Report No. CMS-EXO-16-010. 
[146] G. Aad et al. (ATLAS Collaboration), Search for a heavy charged boson in events with a charged lepton and missing transverse momentum from $p p$ collisions at $\sqrt{s}=13 \mathrm{TeV}$ with the ATLAS detector, Phys. Rev. D 100, 052013 (2019).

[147] K. Park, S. Lee, W. Jun, and U. Min, Re-implementation of the $\mathrm{W}^{\prime}$ into 1 lepton + missing energy analysis $139 \mathrm{fb}^{-1}$, Report No. ATLAS-EXOT-2018-30.

[148] J. Y. Araz, M. Frank, and B. Fuks, Reinterpreting the results of the LHC with MadAnalysis 5: Uncertainties and higherluminosity estimates, Eur. Phys. J. C 80, 531 (2020).

[149] J. Alwall, R. Frederix, S. Frixione, V. Hirschi, F. Maltoni, O. Mattelaer, H.-S. Shao, T. Stelzer, P. Torrielli, and M. Zaro, The automated computation of tree-level and nextto-leading order differential cross sections, and their matching to parton shower simulations, J. High Energy Phys. 07 (2014) 079.

[150] M. Aaboud et al. (ATLAS Collaboration), Measurements of higgs boson properties in the diphoton decay channel with $36 \mathrm{fb}^{-1}$ of $p p$ collision data at $\sqrt{s}=13 \mathrm{TeV}$ with the atlas detector, Phys. Rev. D 98, 052005 (2018).

[151] A. Sirunyan et al. (CMS Collaboration), Measurements of higgs boson properties in the diphoton decay channel in proton-proton collisions at $\sqrt{s}=13 \mathrm{TeV}$, J. High Energy Phys. 11 (2018) 185.

[152] G. Belanger, F. Boudjema, A. Pukhov, and A. Semenov, micromegAs: Version 1.3, Comput. Phys. Commun. 174, 577 (2006).
[153] A. Belyaev, N. D. Christensen, and A. Pukhov, CalcHEP 3.4 for collider physics within and beyond the standard model, Comput. Phys. Commun. 184, 1729 (2013).

[154] G. Belanger, F. Boudjema, J. Fujimoto, T. Ishikawa, T. Kaneko, K. Kato, and Y. Shimizu, Automatic calculations in high energy physics and Grace at one-loop, Phys. Rep. 430, 117 (2006).

[155] K. Griest and D. Seckel, Three exceptions in the calculation of relic abundances, Phys. Rev. D 43, 3191 (1991).

[156] X.-L. Chen and M. Kamionkowski, Three body annihilation of neutralinos below two-body thresholds, J. High Energy Phys. 07 (1998) 001.

[157] Y. Hosotani, P. Ko, and M. Tanaka, Stable Higgs bosons as cold dark matter, Phys. Lett. B 680, 179 (2009).

[158] C. E. Yaguna, Large contributions to dark matter annihilation from three-body final states, Phys. Rev. D 81, 075024 (2010).

[159] T. Binder, T. Bringmann, M. Gustafsson, and A. Hryczuk, Early kinetic decoupling of dark matter: When the standard way of calculating the thermal relic density fails, Phys. Rev. D 96, 115010 (2017).

[160] M. Duch and B. Grzadkowski, Resonance enhancement of dark matter interactions: The case for early kinetic decoupling and velocity dependent resonance width, J. High Energy Phys. 09 (2017) 159. 